\title{
Stability analysis of power amplifiers under output mismatch effects
}

\author{
Almudena Suárez, IEEE Fellow, Franco Ramírez, Sergio Sancho
}

\begin{abstract}
Novel criteria are presented for small- and large-signal stability analysis of microwave circuits under output mismatch effects. They are based on concepts from bifurcation theory, which enable the derivation of general equations for the stability boundaries that depend on the termination load. The boundary calculation does not require any optimization of the circuit parameters. Instead, it is based on the direct calculation of an admittance function obtained through circuit linearization about either the $d c$ solution, in the small-signal stability analysis, or the periodic solution, in the large-signal one, with low computational cost. As will be demonstrated both with homotopy and bifurcation concepts, the Hopf locus obtained in this way delimits a stable region and a potentially unstable one. In a large-signal stability analysis, the sidebands that influence the stability properties are identified, which depend on the particular amplifier topology and its filtering effects. The stability boundaries are then calculated with the conversion matrix approach for all load impedance values at the relevant harmonic and sideband frequencies. For the derivation of absolute stability conditions, a new scattering type matrix is proposed describing the circuit response from the output reference plane with respect to the two sidebands about the input carrier. The new methodologies and formulations have been validated by independent simulations using pole-zero identification, and by measurements.
\end{abstract}

Index Terms - Stability analysis, bifurcation, power amplifier, mismatch effects.

\section{INTRODUCTION}

Power amplifier (PA) designs, which are stable under ideal $50 \Omega$ terminations, may become unstable [1-5] under antenna mismatch conditions in realistic wireless environments, such as where the antenna input impedance is affected by objects in its vicinity [1-8]. Therefore, evaluation of the stability robustness against load mismatches is essential for reliable operation. The works [1-3], based on pole-zero identification, require a systematic test of impedances on the Smith chart and involve a high computational cost. In [9] a new methodology was presented for an efficient calculation of the stability boundaries using concepts from bifurcation theory [10]. The analysis in [9] is applied to a finished circuit design, which should be stable with standard $50 \Omega$ terminations. Note that

This work was supported by the Spanish 'Ministry of Economy and Competitiveness,' under contract TEC2011-29264-C03-01.

Authors are with the Communications Engineering Department, University of Cantabria, Santander, 39005 Spain, (e-mail: suareza@unican.es; ramirezf@unican.es; $\underline{\text { sanchom@unican.es). }}$ the analysis is not carried out at the intrinsic device terminals, but at a reference plane located beyond the output network [9], where the original $50 \Omega$ termination is replaced with a mismatched load $\Gamma_{L}$. This corresponds to the input impedance of the next system block, usually the antenna. Advantage is taken of the fact that at the stability boundary, the steady-state oscillation condition is fulfilled with oscillation amplitude tending to zero $[9,11]$, corresponding to a Hopf bifurcation [12-14]. The analysis in [9] was based on the representation of the Hopf locus in the Smith chart. However, this locus will not always provide a closed boundary, so additional research is necessary to distinguish between stable and unstable regions. Furthermore, no rigorous proof of its validity as a stability boundary under frequency-varying loads $\Gamma_{L}(f)$ was given in [9]. The locus exhibits a well-defined dependence on the oscillation frequency. However, the frequency characteristic $\Gamma_{L}(f)$ of the mismatched antenna is unknown in practice. Using homotopy concepts, it will be rigorously demonstrated here that the Hopf locus traced in the $\Gamma_{L}$ plane distinguishes frequency characteristics with stable and potentially unstable behavior. The new theory will also enable a correct interpretation of the Hopf locus geometry in all cases.

In the large-signal regime, the PA or, in general, the active microwave circuit operates under nonlinear conditions with respect to the input source at the frequency $f_{\text {in }}$ and linearly with respect to the perturbation at the frequency $f$ [15-16]. The analysis in [9] takes into account that the output load will have certain frequency dependence, neglected in previous works [1-3], and therefore, it will exhibit different values at the various frequencies $k f_{\text {in }}$ and $k f_{\text {in }}+f$, where $k$ is an integer [9]. However, as will be rigorously verified in this paper, if the output circuit exhibits a filtering behavior, not all the frequencies $k f_{\text {in }}$ and $k f_{\text {in }}+f$ will have an impact on the circuit stability. In the large-signal regime, Hopf loci are calculated under variation of the relevant harmonic and sideband immittances. In [9] this was done using the conversion matrix approach [15-16] (small-signal/largesignal analysis) of commercial harmonic balance (HB) software. Here a detailed mathematical analysis is presented, enabling deep insight into the effect of mismatched terminations. In the case of two dominant sidebands, this analysis reduces the linearized description of the circuit response to a $2 \times 2$ outer tier impedance/admittance matrix, 
depending on the output load at the fundamental frequency. This matrix will provide the theoretical basis for the generalization of Rollet's stability criteria and stability circles [27] to large-signal regimes under output mismatch effects.

An advantage of the new method derives from the fact that, at the Hopf bifurcation, the circuit is in a limit steady-state oscillation condition [11], so each Hopf bifurcation can be accurately calculated using a discrete set of load impedance values at the relevant harmonic and sideband frequencies, at the particular oscillation frequency $f_{0}$. This is different from regular stability analyses that require knowledge of the frequency response of all the circuit elements [17-25]. Two different situations are analyzed in two nonlinear amplifiers used as demonstrators. One of them corresponds to instabilities already existing in the small-signal regime, which are extinguished above a certain input power (asynchronous extinction) [11]. The other, of great relevance in PAs, corresponds to circuits that are originally stable in the smallsignal regime, but which become unstable from certain input power [26]. In future work, we will consider conditions as close as possible to real situations found in practical applications

As already stated, this work will present an extension of the absolute stability criteria and stability circle theory derived by Rollet [27] to circuits in large-signal periodic regime, under output mismatch effects. The formulation considers two relevant sidebands, usually the lower and upper sideband of the input frequency $\left(f_{\text {in }}-f\right.$ and $\left.f_{\text {in }}+f\right)$, for particular values of the termination impedances at the rest of analysis frequencies. It is based on the derivation of a $2 \times 2$ linear matrix, describing the linearized circuit response from its output terminals, at the two sidebands. The output terminals correspond to those at which the antenna or the next system component is connected. For given terminations at $k f_{\text {in }}$ and $k f_{\text {in }}+f($ with $|k| \neq 1$ ), the $2 \times 2$ matrix is easily obtained using commercial software via the conversion matrix approach. Using this matrix, absolute stability criteria in large-signal regime are derived, which in the general case should be evaluated for each set of impedance values at the rest of the analysis frequencies. A higher order matrix would be derived in the case of more than two relevant sidebands. Then, existing generalizations [28-29] of Rollet's theory to more than two physical ports could be adapted to this matrix, at the expense of a higher computational cost. Under conditional stability, an extension of the stability circle concept will provide the boundary of impedance terminations at one sideband $f_{\text {in }}+f$ (or $f_{\text {in }}-f$ ) giving rise to negative resistance at the other sideband $f_{\text {in }}-f\left(\right.$ or $\left.f_{\text {in }}+f\right)$. As will be shown, stability circles obtained in this manner can be used for an efficient calculation of the Hopf loci.

To demonstrate the generality of the method, it has been applied to two different amplifiers. The results have been validated using various passive loads, which have enabled a comparison with well-established analysis techniques, such as pole-zero identification [21-25] or bifurcation detection with auxiliary generators $[11,14,26]$. Measurements have also been carried out for the two amplifiers, which exhibited unstable behavior under the conditions predicted by the method.

Section II presents the small-signal stability analysis with a detailed study of the geometry and properties of the Hopf bifurcation locus. Section III describes the large-signal stability analysis with the novel methodology that traces the Hopf locus using the conversion matrix approach. Section IV introduces the $2 \times 2$ scattering type matrix used for the analysis of conditional/unconditional stability. It also details the use of stability circles (negative resistance boundaries) to obtain the Hopf bifurcation loci in an efficient manner.

\section{SMALL-SignAl ANALYSIS}

It is assumed that the circuit is stable with an ideal $50 \Omega$ termination (Fig. 1), though it can become unstable for particular loading conditions (conditional stability). It is also assumed that it fulfills the Rollet proviso, that is, it does not contain any poles on the right-hand side of the complex plane when the termination loads are replaced with zero or infinite impedances [18-19,27]. Indeed, the objective is to analyze the possible destabilization due to output mismatch effects. With this aim, the stable $50 \Omega$ output load will be replaced with a variable one, represented by the reflection coefficient $\Gamma_{L}=\rho \mathrm{e}^{j \theta}$ (Fig. 1). For the stability analysis under smallsignal conditions, the input source is set to zero and only the perturbation frequency $f$ is considered.

In a steady-state oscillation, the total admittance will be equal to zero at all the circuit nodes [30]. An analogous condition can be derived in terms of impedances. In particular, at the stability boundary, the above steady-state oscillation condition is fulfilled for oscillation amplitude tending to zero $[11,14]$. This corresponds to a Hopf bifurcation $[11,14]$ or oscillation generated from a $d c$ regime. As all the nodes fulfill the oscillation condition, the zero-amplitude oscillation (at the stability boundary) can be analyzed at the circuit output node (Fig. 1, without the input source). As derived in [9], denoting the output load admittance as $Y_{L}$, the Hopf bifurcation condition (in both the supercritical and subcritical case) is:

$$
Y_{\text {out }}\left(f_{o}\right)+Y_{L}=Y_{\text {out }}\left(f_{o}\right)+Y_{c} \frac{1-\rho e^{j \theta}}{1+\rho e^{j \theta}}=H\left(f_{o}, \rho, \theta\right)=0
$$

where $Y_{\text {out }}$ is the circuit admittance looking into the circuit from the output terminals (Fig. 1), $Y_{C}$ is the reference admittance and $f_{o}$ the oscillation frequency at the stability boundary. Equation (1) defines a Hopf bifurcation locus $[11,14]$ in the plane $\rho, \theta$. The output admittance $Y_{\text {out }}(f)$ is calculated with a simple small-signal frequency sweep, and the reflection coefficient at the stability boundary is determined from (1).

To compare, Rollet's analysis provides the boundary (stability circle) of source- (load-) impedance values of a two-port network that give rise to negative resistance when looking into the output (input) port of the network [31]. 
Equation (1) is applied to a finished design and considers the effect of replacing the original $50 \Omega$ termination with unknown impedance coming from the mismatched antenna. Unlike Rollet's analysis, which predicts the source/load impedances giving rise to negative resistance, (1) is an actual oscillation condition with amplitude tending to zero.

The analysis in (1) has been applied to the power amplifier in Fig. 1 that is based on the transistor CLY5 (Triquint - High power GaAs FET) biased at $V_{G G}=-2.14 \mathrm{~V}$ and $V_{D D}=3 \mathrm{~V}$. The Hopf locus starts near an open circuit at low frequencies, and ends up near a short circuit at high frequencies after one and a half turns around the origin of the Smith chart (Fig. 2). When using $\rho$ and $\theta$ as frequency independent parameters, the intersection of the Hopf locus with the unit Smith chart delimits the oscillation and non-oscillation regions.

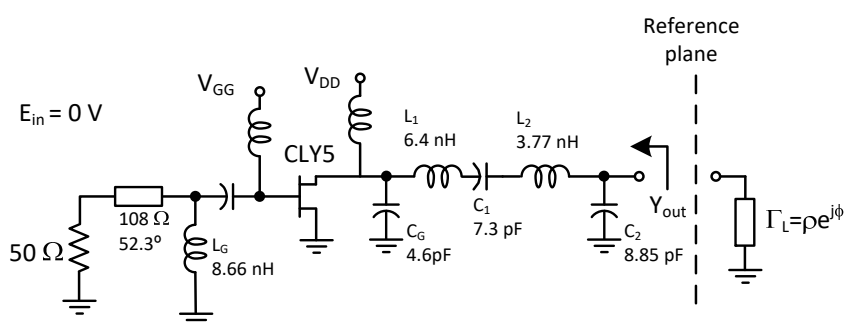

(a)

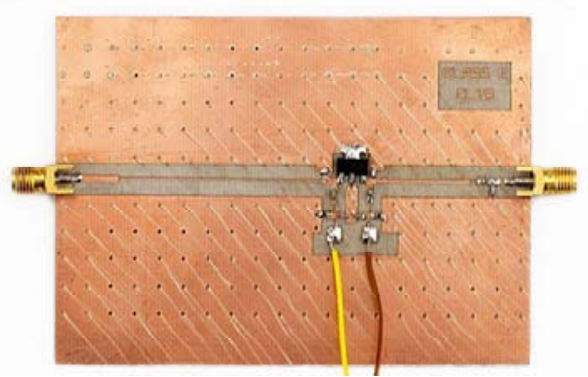

(b)

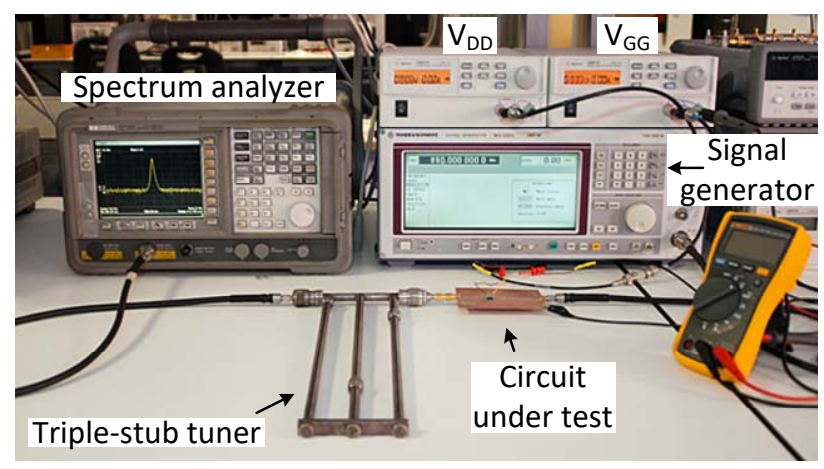

(c)

Fig. 1 (a) Schematic of a PA with $f_{\text {in }}=0.8 \mathrm{GHz}, V_{G G}=-2.14 \mathrm{~V}$, $V_{D D}=3 \mathrm{~V}$, and efficiency of $55 \%$ at $P_{\text {out }}=27 \mathrm{dBm}$ when loaded with $50 \Omega$. (b) Photograph of PA circuit. For the analysis under mismatch effects, the $50 \Omega$ output load is replaced with a general load described by the reflection coefficient $\Gamma_{L}$. (c) Photograph of the measurement setup. A triple-stub tuner is connected at the output of the amplifier to consider different $\Gamma_{\mathrm{L}}$ values.

The Hopf boundary is inside the unit Smith chart in the critical frequency interval from $f_{1}=651 \mathrm{MHz}$ to $f_{2}=820 \mathrm{MHz}$.
Circuit oscillation should occur at frequencies in the interval $\left(f_{1}, f_{2}\right)$. This is because the fulfillment of oscillation conditions becomes more demanding as the amplitude increases due to the associated physical reduction of negative resistance. This is demonstrated as follows.

Defining $Y_{\text {out }}$ as an outer-tier admittance function, including both linear and nonlinear elements, this function will depend on both the excitation amplitude $A$ and frequency $f$, that is, $Y_{\text {out }}=Y_{\text {out }}(A, f)$. In order to fulfill (1) at small signal, $\operatorname{Re}\left[Y_{\text {out }}\left(A=0, f_{o}\right)\right]$ must be negative. The increment of the admittance function with respect to the small-signal value will be calculated as: $\Delta Y_{\text {out }}=Y_{\text {out }}(A, f)-Y_{\text {out }}(A=0, f)$. After the Hopf bifurcation, when the $d c$ solution is unstable, we will have $\operatorname{Re}\left[\Delta Y_{\text {out }}(A, f)\right]>0$. This will be fulfilled after both supercritical and subcritical bifurcations $[10-12,32]$. Restricting the analysis to passive loads $\left[\operatorname{Re}\left(Y_{L}(f)>0\right]\right.$, the following steady-state oscillation conditions are satisfied:

$$
\begin{aligned}
& \operatorname{Re}\left[Y_{\text {out }}\left(A, f_{o}\right)+Y_{L}\right]=\operatorname{Re}\left[Y_{T S s}\left(0, f_{o}\right)\right]+\operatorname{Re}\left[\Delta Y_{\text {out }}\left(A, f_{o}\right)\right]=0 \\
& \operatorname{Im}\left[Y_{\text {out }}\left(A, f_{o}\right)+Y_{L}\right]=\operatorname{Im}\left[Y_{\text {Tss }}\left(0, f_{o}\right)\right]+\operatorname{Im}\left[\Delta Y_{\text {out }}\left(A, f_{o}\right)\right]=0
\end{aligned}
$$

where $Y_{\text {TsS }}$ refers to the total admittance under small-signal conditions evaluated using (1). Because $\operatorname{Re}\left[\Delta Y_{\text {out }}(A, f)\right]$ is positive, (2a) is necessarily fulfilled for a smaller frequency interval than (1), regardless of the particular form of the frequency function $Y_{\text {out }}(A, f)$.

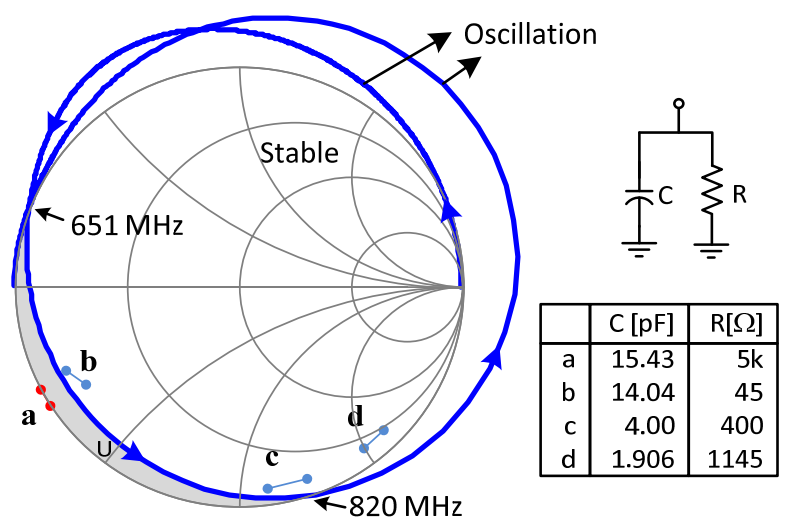

Fig. 2 Stability boundary under small-signal regime. The $\Gamma_{\mathrm{L}}(f)$ functions corresponding to different output load networks (a, b, c, d) have been represented.

The reduction of the critical frequency interval with the steady-state oscillation amplitude is confirmed with the auxiliary generator (AG) technique [11]. The steady-state periodic oscillation (in the absence of input drive) is analyzed by loading the fundamental frequency $f_{\mathrm{o}}$ with $\Gamma_{L}=\rho e^{j \theta}$ and the harmonic terms with $50 \Omega$. Note that the harmonic termination should have little relevance in this case due to the action of the output filter. A voltage $A G$, with amplitude $A_{A G}$, is connected in parallel to the drain node. The AG must fulfill 
the non-perturbation condition given by the zero value of the ratio between the current circulating through the $\mathrm{AG}$ and the voltage delivered, that is, $Y_{A G}=0$ [11]. To obtain the contours of constant $A_{A G}$, the phase of the output load reflection coefficient $\theta$ is swept, solving the non-perturbation condition in terms of the reflection coefficient magnitude $\rho$ and the oscillation frequency $f_{o}$ at each $\theta$ value. The contours with increasing oscillation amplitudes (from $A_{A G}=0.5 \mathrm{~V}$ to $A_{A G}=0.8 \mathrm{~V}$ ) are shown in Fig. 3. For $A_{A G}=0.5 \mathrm{~V}$ the oscillation frequency interval is $706 \mathrm{MHz}$ to $817 \mathrm{MHz}$. For $A_{A G}=0.8 \mathrm{~V}$, the oscillation frequency interval is $774 \mathrm{MHz}$ to $806 \mathrm{MHz}$. These results confirm the reduction of the oscillation frequency interval with the steady-state oscillation amplitude. The study indicates that each particular $\Gamma_{L}=\Gamma_{L o}$ within the "U" region would give rise to an oscillation of given amplitude $A_{o}$ and frequency $f_{o}$. Note that the oscillation analysis in Fig. 3 assumes frequency independent loads. With a realistic frequency varying load $\Gamma_{L}(f)$, such an oscillation (with amplitude $A_{o}$ and frequency $f_{o}$ ) would be obtained if this load fulfills $\Gamma_{L}\left(f_{o}\right)=\Gamma_{L o}$. Note that the frequency characteristic $\Gamma_{L}(f)$ is not known in advance. Therefore, " $U$ " is a region of potential oscillation.

Solely with the representation of the Hopf locus in the Smith chart, as was done in [9], it may be difficult to distinguish between stable and unstable load values. For instance, in the chart in Fig. 2, there are open regions on the right-hand side of the unit Smith chart that are not separated by any portion of the Hopf locus, acting as a border or boundary between regions with different stability properties. As will be shown, the full distinction between the stable and unstable zones will require an "auxiliary" representation of the Hopf locus in the plane defined by amplitude and phase of the load reflection coefficient $\rho$ and $\theta$, which are the actual analysis parameters in (1). The fact that this Hopf locus constitutes a stability boundary in the presence of frequencyvarying loads $\Gamma_{L}(f)$ will be demonstrated here for the first time using homotopy methods.

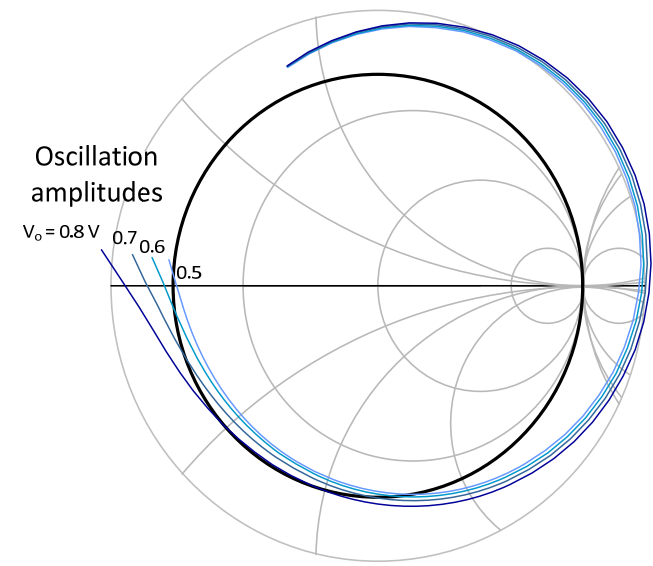

Fig. 3 Loci of oscillation points with different steady-state amplitudes $A_{A G}=0.5 \mathrm{~V}$ to $A_{A G}=0.8 \mathrm{~V}$. All of them are included in the unstable region delimited by the Hopf locus (Fig. 2).
To illustrate the demonstration, the Hopf locus corresponding to the circuit in Fig. 1 has been traced in the plane defined by $\rho$ and $\theta$ [Fig. 4(a)]. The phase has been unwrapped and it goes beyond $360^{\circ}$ since the oscillation frequency changes along the plot and is implicit in the representation. In fact, the same constant value $\Gamma_{L}=\rho \mathrm{e}^{\mathrm{j} \theta}$ can give rise to Hopf bifurcations at two different frequencies, which explains the self-intersection of the Hopf locus in Fig. 2. In this particular case, non-oscillatory solutions are obtained on the left-hand side of the Hopf locus. Oscillatory solutions [in terms of $\rho, \theta$ as explained above] are on the right side of this locus. In the Smith chart representation of Fig. 2, oscillations are obtained in the sense of the arrows. In each practical application, once the geometry of the oscillation region has been clarified, there is no need to use the auxiliary $\rho-\theta$ plane.

For compactness, the Hopf locus obtained with (1) will be denoted $\Gamma_{H}\left(f_{o}\right) \equiv \rho\left(f_{o}\right) e^{j \theta\left(f_{o}\right)}$. Geometrically, the Hopf bifurcation locus will delimit two regions $A_{1}$ and $A_{2}$ in the $\Gamma_{L}$ plane [Fig. 4(a)]. In particular, we will assume that the stable load $\Gamma_{L}=0$ belongs to region $A_{1}$. In the following, this region will be demonstrated to be stable. Let the curve associated with $\Gamma_{H}(f)$ be denoted $C\left(\Gamma_{H}\right)$ and the curve associated with $\Gamma_{L}(f)$ be denoted by $C\left(\Gamma_{L}\right)$. The small-signal solution of the circuit loaded with $\Gamma_{L}(f)$ will have a pair of complexconjugate poles in the imaginary axis, $\pm j 2 \pi f_{o}$, if and only if the curves $C\left(\Gamma_{L}\right)$ and $C\left(\Gamma_{H}\right)$ intersect at the particular frequency $f_{o}$, that is:

$$
\exists f_{o} \in \Re \mid \Gamma_{L}\left(f_{o}\right)=\Gamma_{H}\left(f_{o}\right)
$$

Note that it is sufficient to evaluate the above condition in the critical frequency interval $F_{c}$. Let a particular load $\Gamma_{L}(f)$, remaining in $A_{1}$ for the whole interval $F_{c}$ be considered. The corresponding admittance curve, traced in $F_{c}$ only, will be denoted by $C\left(\Gamma_{L}, F_{c}\right)$. In order to define a homotopy transformation [10], one or more parameters $\bar{\eta}$ will be introduced in $\Gamma_{L}(f)$, obtaining the function $\Gamma_{L}(f, \bar{\eta})$. These parameters $\bar{\eta}$ may be either physical or artificial. A pair of initial and final values $\left(\bar{\eta}_{o}, \bar{\eta}_{f}\right)$ can be chosen such that $\Gamma_{L}\left(f, \bar{\eta}_{o}\right)=\Gamma_{L}(f)$ and $\Gamma_{L}\left(f, \bar{\eta}_{f}\right)=0$. Under the continuous variation of $\bar{\eta}$, the curve traced in $F_{C}$ only will evolve as $\mathrm{C}\left(\Gamma_{L}, F_{c}, \bar{\eta}\right)$. Because both $\mathrm{C}\left(\Gamma_{L}, F_{c}\right)$ and the stable load $\Gamma_{L}=0$ belong to the same region $A_{1}$, the curve can be continuously deformed through an infinite set of differential transformations without intersecting the Hopf locus $\mathrm{C}\left(\Gamma_{H}, F_{C}\right)$ until reaching $\Gamma_{L}=0$. This property, together with the fact that the circuit is structurally stable [10] when loaded with $\Gamma_{L}=0$, guarantee that $\Gamma_{L}(f)$ is a stable load. 


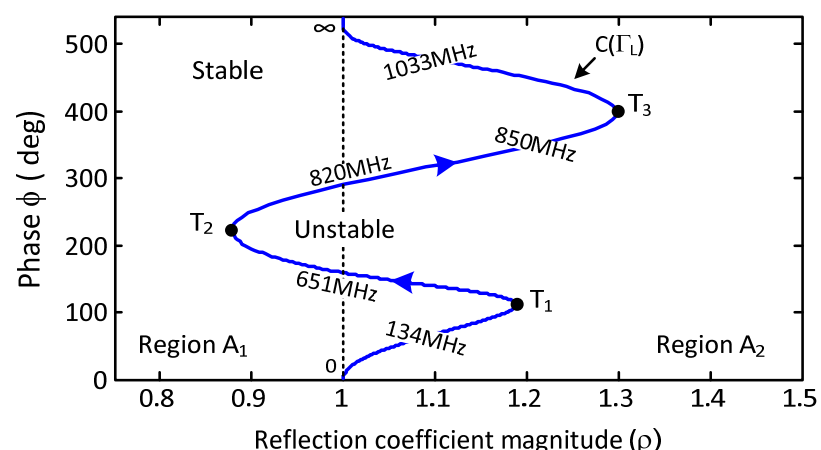

(a)

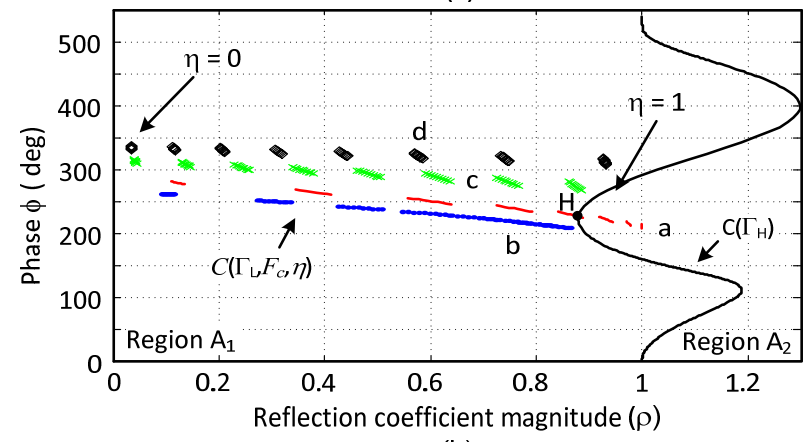

(b)

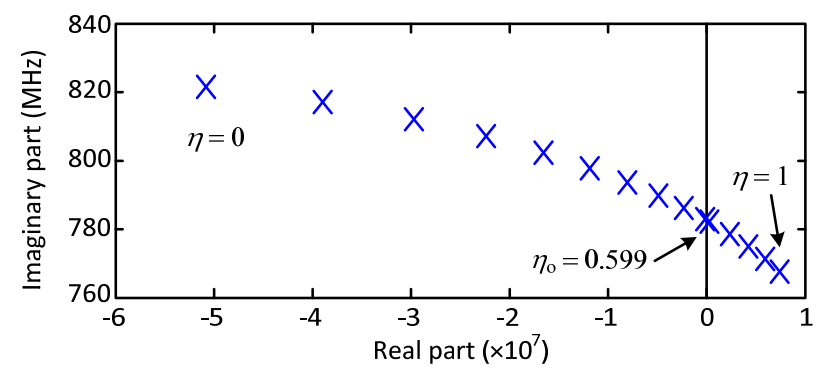

(c)

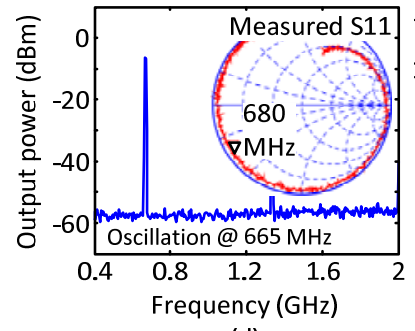

(d)

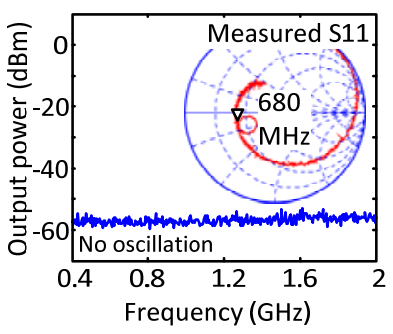

(e)
Fig. 4 Hopf bifurcation locus of the power amplifier in Fig. 1 in the plane defined by $\rho$ and $\theta$. (a) Hopf locus. The circuit oscillates on the right-hand side of this locus. (b) Evolution of the four loads in Fig. 2, evaluated in $F_{C}$ when reducing $\eta$ in (4) from 1 to 0 in 0.1 steps. (c) Validation using pole-zero identification. The complex-conjugate poles cross the imaginary axis at the same parameter value $\eta_{o}=0.599$ and perturbation frequency $782.7 \mathrm{MHz}$ predicted by the homotopy transformation. (d) Spectrum measurement for a load in $A_{2}$ (e) Spectrum measurements for a load in $A_{1}$.

As an example, Fig. 2 shows the frequency response of four different $\Gamma_{L}$ loads obtained through the parallel connection of a resistor and a capacitor ( $\mathrm{RC}$ loads). The four loads are evaluated in the critical frequency interval $f_{1}$ to $f_{2}$. In case "a" [see Fig. 4(b)], the impedance of the load network enters the unstable region. Therefore, the circuit with this particular load is likely to be unstable. In the other three cases "b", "c" and "d", the reflection coefficient remains on the stable side. Just for illustration, homotopy transformations will be applied to these four loads. A parameter $\eta$ is artificially introduced in the admittance function:

$$
Y_{L}(f, \eta)=(1-\eta) G_{o}+\eta[G+\mathrm{j} 2 \pi f C]
$$

where $G_{o}=1 / 50 \mathrm{~S}, G=1 / R$. For each network, at $\eta=1$, one has the original RC load. On the other hand, at $\eta=0$, the load becomes $G_{o}=1 / 50 \mathrm{~S}$, so the circuit is necessarily stable. The evolution of the four original loads when reducing $\eta$ from 1 towards 0 in 0.1 steps is shown in Fig. 4(b). Only load "a" crosses the Hopf locus in the critical frequency interval. The condition $\Gamma_{L}\left(f_{o}\right)=\Gamma_{H}\left(f_{o}\right)$ is fulfilled at point $\mathrm{H}$, which means that there will be a qualitative change of stability at this point. The intersection point $\left(f_{o}, \eta_{o}\right)$ can be easily obtained by minimizing the function:

$$
d(f, \eta)=\left|\Gamma_{L}(f, \eta)-\Gamma_{H}(f)\right|^{2}
$$

The crossing takes place at $f_{o}=782.7 \mathrm{MHz}, \eta_{o}=0.599$. This result has been validated with pole-zero identification [21$23,25]$. The identification is performed introducing a smallsignal current source in parallel at the transistor drain terminal and calculating a closed-loop transfer function, given by the ratio between the node voltage and the current introduced. As shown in Fig. 4(c), the complex-conjugate poles cross the imaginary axis at the same parameter value $\eta_{0}=0.599$ and perturbation frequency $782.7 \mathrm{MHz}$ predicted by the homotopy transformation. Measurements have been carried out using a triple-stub tuner, as depicted in Fig. 1c. The output spectra for particular loads in Region $A_{2}$ and Region $A_{1}$ are shown in Fig. 4(d) and Fig. 4(e). Pole-zero identification [21-23,25] has also been used to validate the predictions of the Hopf bifurcation locus using the load networks in Fig. 2 (Verification I). As shown in Fig. 5(a), the circuit is unstable in case "a", when there is a pair of poles at $790 \mathrm{MHz}$ [belonging to the critical interval $\left.\left(f_{1}, f_{2}\right)\right]$ on the right-hand side of the complex plane. In case "b", the load impedance does not enter the unstable region but it is very close to this region. The circuit is stable [Fig. 5(b)], with the pair of poles close to the imaginary axis. For the other two loads ("c" and "d") in Fig. 2, the circuit is also stable.

If the frequency characteristic of the mismatched load could be measured or modeled, this can be combined with the output circuit admittance $Y_{\text {out }}(f)$ to obtain an impedance type closed-loop transfer function [21-23,25] given by:

$$
H(f)=\frac{1}{Y_{\text {out }}(f)+Y_{L}(f)}
$$

The analysis of this $H(f)$ is equivalent to that of a closedloop transfer function obtained by connecting a small-signal 
current source in parallel at the output node. The amplitude and phase of $H(f)$ can be represented to predict possible unstable resonances. The results should be equivalent to those of a Bode analysis [33-34]. Because the possible instability would be due to output mismatch effects, one can expect this instability to be detectable from the circuit output terminals. In the case of load "a", the positive slope of the phase characteristic at the resonance frequency [Fig. 5(c)] indicates unstable behavior, in agreement with the results of the polezero identification [21-23,25] in [Fig. 5(a)]. In the case of load "b" [Fig. 5(d)], the slope of the phase is negative, in total agreement with the results of the pole-zero identification in [Fig. 5(b)].

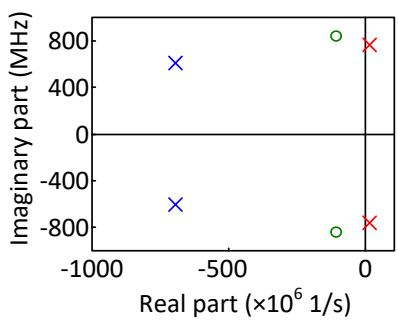

(a)

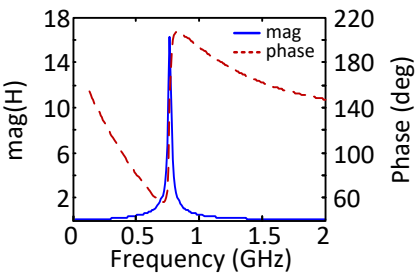

(c)

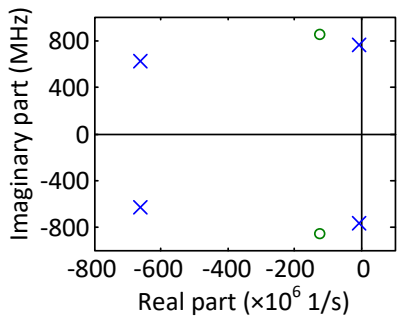

(b)

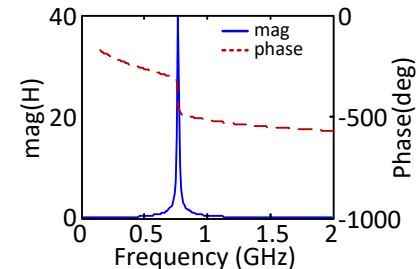

(d)
Fig. 5 Validation of the stability boundary with pole-zero identification. (a) Unstable behavior for load "a" in Fig. 2. (b) Stable behavior for load "b". (c) Transfer function, calculated with (6), corresponding to "a". (d) Transfer function corresponding to " $b$ ".

\section{LARGE-SignAl StABILITy AnAlysis AgAinst Mismatch EFFECTS}

As in a small-signal case, it will be assumed that the circuit is stable with the $50 \Omega$ output load in the large signal analysis. Moreover, with this $50 \Omega$ load, it must be stable for all input power values, with instability occurring only due to mismatch effects. For the large-signal stability analysis, one must take into account the sidebands generated by the perturbation frequency $f$ about the spectral lines of the steady-state regime, that is, $k f_{\text {in }}+f$, where $-N \leq k \leq N$ and $N$ is the number of harmonic terms considered. The circuit behaves nonlinearly with respect to the input source at $f_{\text {in }}$ and linearly with respect to the perturbation at $f$. In general, the output matching network will have a filtering effect. Therefore, it will be possible to limit the stability analysis to only a few of the sidebands $k f_{\text {in }}+f$. This is because the analysis is not carried out at the intrinsic device terminals (where all the spectral lines will have an effect on the stability properties), but at a reference plane located beyond the circuit output filter, which prevents the influence of most of the sidebands. For instance, in the amplifier in Fig. 1, due to the high-frequency selectivity of the output network, the stability analysis can be limited to $f_{l}=f_{\text {in }}-f, f_{\text {in }}$ and $f_{u}=f_{\text {in }}+f$. Mismatch effects at the baseband frequency $f$ and sideband frequencies $k f_{\text {in }}+f$, with $|k|>1$, will not have any impact on the stability properties. The fulfillment of this assumption will be verified in all cases. Perturbation frequency variations in the interval $0<f<f_{\text {in }} / 2$ will be considered. This reduction of the analyzed frequency interval is enabled by the pole repetition with periodicity $f_{\text {in }}$ inherent in the stability of periodic solutions, and due to the non-univocal relationships between the Floquet multipliers and the poles [23]. Furthermore, due to the action of the output filter, this will be the frequency interval with maximum observability.

In the following, we present a detailed demonstration of the analysis methodology applied "empirically" in [9], through direct use of the small-signal large-signal analysis of the HB software. The demonstration will provide the theoretical basis for a newly defined $2 \times 2$ scattering type matrix, enabling the generalization of Rollet's analysis to large signal periodic regimes under output mismatch effects.

In the two PA examples considered in this work, only termination at the three frequencies $f_{l}, f_{\text {in }}$ and $f_{u}$ are relevant. The load reflection coefficients at $f_{l}, f_{\text {in }}$ and $f_{u}$ are $\Gamma_{l}, \Gamma_{o}$ and $\Gamma_{u}$, respectively (Fig. 6). At all other frequencies, the original termination $\left(\Gamma_{L}=0\right)$ is considered for simplicity. That is:

$$
\begin{aligned}
& f_{\text {in }} \rightarrow \Gamma=\Gamma_{\mathrm{o}} \\
& |k| f_{\text {in }},|k| \neq 1 \rightarrow \Gamma=0 \\
& f_{\text {in }}-f \rightarrow \Gamma=\Gamma_{l} \\
& f_{\text {in }}+f \rightarrow \Gamma=\Gamma_{u} \\
& |k| f_{\text {in }} \pm f,|k| \neq 1 \rightarrow \Gamma=0
\end{aligned}
$$

The above load values (except $\Gamma_{l}$ ) are implemented using ideal filters and ideal loads in commercial software. A sketch is shown in Fig. 6.

The reflection coefficient $\Gamma_{o}$ affects the steady-state solution, and therefore, the periodic regime about which the circuit is linearized. This dependence of the steady-state solution can be expressed in a compact manner as:

$$
\bar{H}\left(\bar{X}_{o}, \Gamma_{o}, E_{\text {in }}\right)=0
$$

where $\bar{H}$ is the vector of error functions of the HB equations, $\bar{X}_{o}$ is the vector of the harmonic components of the steadystate solution and $E_{\text {in }}$ is the input generator amplitude. For the calculation of the Hopf bifurcation loci, a single analysis port is considered looking into the circuit output (see Fig. 6). Thus, it will be possible to model the linearized circuit with an admittance matrix, relating the output port current and voltage components at all the sideband frequencies $k f_{\text {in }}+f$ :

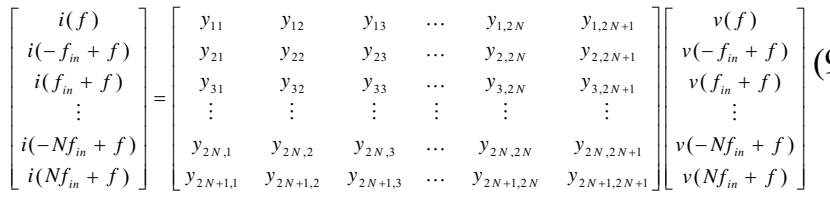


where $N$ is the number of harmonic terms. The above matrix inherently depends on the circuit linearization about the steady-state regime performed with the conversion matrix approach.

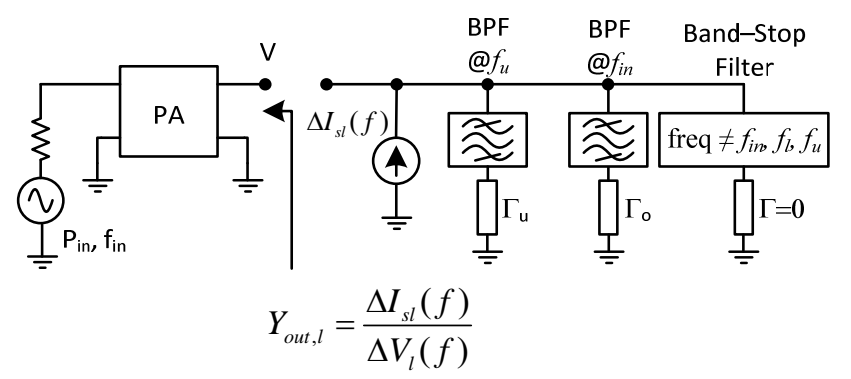

Fig. 6 Arrangement of ideal filters and ideal loads used to ensure different $\Gamma_{L}$ values at the various harmonic and sideband frequencies according to (7). The small-signal current source is used to calculate the input admittance at the lower frequency sideband $f_{l}$.

As already stated, in this analysis, it will be assumed that all the sidebands $k f_{\text {in }}+f$ with $k$ different from \pm 1 are terminated with the reference impedance $Z_{c}$, although, due to the filter action it would be equally possible to consider any other termination impedances. Then, it is possible to add to (9) $2 N-1$ equations of the form:

$$
i\left(k f_{\text {in }}+f\right)=-\frac{1}{Z_{c}} v\left(k f_{\text {in }}+f\right), \quad \text { for } \quad|k| \neq 1
$$

Combining (9) and (10), one obtains a reduced system in the two sideband frequencies of interest:

$$
\begin{aligned}
& \Delta I_{l}^{*}(f)=Y_{l, l} \Delta V_{l}^{*}(f)+Y_{l, u} \Delta V_{u}(f) \\
& \Delta I_{u}(f)=Y_{u, l} \Delta V_{l}^{*}(f)+Y_{u, u} \Delta V_{u}(f)
\end{aligned}
$$

where the four parameters $Y_{l, l}, Y_{l, u}, Y_{u, l}, Y_{u, u}$ depend on the elements of the matrix in (9). The following change of notation has been carried out for simplicity:

$$
\begin{array}{ll}
\Delta V_{l}^{*}(f)=V\left(-f_{\text {in }}+f\right), & \Delta V_{u}(f)=V\left(f_{\text {in }}+f\right) \\
\Delta I_{l}^{*}(f)=I\left(-f_{\text {in }}+f\right), & \Delta I_{u}(f)=I\left(f_{\text {in }}+f\right)
\end{array}
$$

To obtain an equation similar to (1) providing the oscillation boundary, a small-signal current source $\Delta I_{s l}(f)$ at $f_{l}=f_{\text {in }}-f$ is connected in parallel at the output node (see Fig. 6), The termination load at the upper sideband will be the complex passive admittance $Y_{L u}$ (corresponding to the reflection coefficient $\Gamma_{u}$ ). Then, the outer-tier circuit equations at the two sideband frequencies $f_{l}$ and $f_{u}$ can be written as:

$$
\left\{\left[\begin{array}{cc}
Y_{l, l}(f) & Y_{l, u}(f) \\
Y_{u, l}(f) & Y_{u, u}(f)
\end{array}\right]+\left[\begin{array}{cc}
0 & 0 \\
0 & Y_{L u}
\end{array}\right]\right\}\left[\begin{array}{c}
\Delta V_{l}^{*} \\
\Delta V_{u}
\end{array}\right]=\left[\begin{array}{c}
\Delta I_{s l}^{*} \\
0
\end{array}\right]
$$

The output admittance at the lower sideband frequency is given by the ratio $Y_{\text {out }, l}=\Delta I_{s l}(f) / \Delta V_{l}(f)$ directly obtained from (13):

$$
Y_{\text {out }, l}^{*}=-\frac{Y_{l, u}(f) Y_{u, l}(f)}{Y_{u, u}(f)+Y_{L u}}+Y_{l, l}(f)
$$

As shown by (14), the output admittance at the lower sideband frequency, assumed to agree with the oscillation frequency, depends on the circuit linearized response [circuit output admittance matrix in (13)] and the load admittance termination at the upper sideband $Y_{L u}$. Note that a similar conversion matrix reduction has been carried out in the works by G. Leuzzi, et al. [35-36] for the analysis/synthesis of frequency divisions. For the frequency division by two, a specific conversion-matrix formulation had to be used, leading to a single complex component at $f_{\text {in }} / 2$. Unlike the methodology presented here, data extracted from $\mathrm{HB}$ had to be complemented with in-house software, as indicated in [36].

The oscillation condition can equally be written at the upper sideband. In an analogous manner, a small-signal current source $\Delta I_{s u}(f)$ would be introduced at $f_{u}=f_{\text {in }}+f$, with the lower sideband terminated with $Y_{L l}$, which provides:

$$
\left\{\left[\begin{array}{cc}
Y_{l, l}(f) & Y_{l, u}(f) \\
Y_{u, l}(f) & Y_{u, u}(f)
\end{array}\right]+\left[\begin{array}{cc}
Y_{L l}^{*} & 0 \\
0 & 0
\end{array}\right]\right\}\left[\begin{array}{l}
\Delta V_{l}^{*}(f) \\
\Delta V_{u}(f)
\end{array}\right]=\left[\begin{array}{c}
0 \\
\Delta I_{s u}(f)
\end{array}\right]
$$

System (13) and system (15) lead to the same results, which is immediately demonstrated by substituting $Y_{L l}=-Y_{\text {out }, l}$ into (15). This replacement directly provides $Y_{o u t, u}=-Y_{L u}$. Therefore, it is sufficient to consider only one of the two analyses at either the lower or the upper sideband. Without loss of generality, the lower sideband analysis (14) will be considered here.

The reflection coefficient $\Gamma_{l}$ at each Hopf bifurcation point is obtained in a manner similar to (1), using the following equation:

$$
Y_{T}\left(\bar{X}_{o}\left(E_{\text {in }}, \Gamma_{o}\right), \Gamma_{u}, \Gamma_{l}, f\right)=Y_{\text {out }}\left(\bar{X}_{o}\left(E_{\text {in }}, \Gamma_{o}\right), \Gamma_{u}, f\right)+Y_{c} \frac{1-\Gamma_{l}}{1+\Gamma_{l}}=0
$$

Equation (16) emphasizes the fact that $Y_{\text {out }}$ at the lower sideband frequency depends on the steady-state solution $\bar{X}_{o}$ obtained at the input amplitude $E_{\text {in }}$, as well as the upper sideband frequency termination $\Gamma_{u}$. Note that derivation (9) to (14) is used for explanatory purposes only, as there is no need to actually perform the calculation of the reduced order $2 \times 2$ matrix representing the circuit admittance from the output terminals at the two sideband frequencies $f_{l}$ and $f_{u}$. In practice, for each pair of reflection coefficient values $\Gamma_{o}, \Gamma_{u}$, the Hopf locus is directly obtained from (16) using the small-signal / large-signal analysis of commercial HB software, as was done in the previous work [9]. A simple $f$ sweep (sketched in Fig. 6) is carried out without any optimization procedures. For each pair of $\Gamma_{o}, \Gamma_{u}$ values, the frequency $f$ is swept between 0 
and $f_{\text {in }} / 2$, solving the complex (16) for the complex variable $\Gamma_{l}$. This provides a curve in the $\Gamma_{l}$ plane for each pair $\Gamma_{o}, \Gamma_{u}$.

The collection of Hopf bifurcation loci obtained in this manner will contain all the possible points $\left[f, \Gamma_{l}(f), \Gamma_{o}(f), \Gamma_{u}(f)\right]$ fulfilling the Hopf bifurcation condition in the circuit terminated with unknown loads. From the results of Section II, if the circuit exhibits Hopf bifurcations, it will necessarily be unstable for particular values of the output load, since an incremental variation of the output load (in particular directions) will lead the critical poles (which are on the imaginary axis at the Hopf bifurcation) to the right-hand side of the complex plane. As the amplifier is stable when loaded with $50 \Omega$, one can conservatively consider that the unstable region is delimited by the envelope of Hopf loci points that are closer to the center of the Smith chart. No attempt is made to obtain stability regions in the high dimension of the parameter space $\Gamma_{l}, \Gamma_{o}, \Gamma_{u}$. However, the influence of $\Gamma_{o}$ and $\Gamma_{u}$ can be easily analyzed through nested sweeps. In general, the dangerous intervals of amplitude and phase are easily identified after a few coarse sweeps. Then, finer sweeps in smaller intervals are carried out.

Note that the same kind of analysis could be applied to detect potential instabilities leading to a division by 2 of the input drive frequency $f_{\text {in. }}$. This may occur in the case of an output network with broader bandwidth about the input frequency. In that case, the small-signal current source should be at $f_{\text {in }} / 2$. To calculate each locus, the phase of this current source should be swept between $0^{\circ}$ and $180^{\circ}$ for each combination of reflection coefficient values at $f_{\text {in }}$ and $3 f_{\text {in }} / 2$. This much simpler analysis is not carried out here since the amplifiers considered did not exhibit this kind of instability. In the free-running oscillator case, the analysis would be carried out using an AG [11] at the oscillation frequency $\omega_{A G}=\omega_{0}$. For each set of termination impedances at the harmonic frequencies, this voltage generator, with amplitude $A_{A G}$, must fulfill the non-perturbation condition $Y_{A G}\left(A_{A G}, \omega_{A G}\right)=0$, with the pure $\mathrm{HB}$ system as the inner tier. In this manner, changes in the oscillation frequency due to antenna mismatch would be taken into account. The effect of the termination impedances at the sideband frequencies could then be analyzed as described in this section and next (Section IV).

The Hopf bifurcation analysis has been applied to the amplifier in Fig. 1. At each input power $P_{i n}$, the Hopf loci obtained with (16) for all possible combinations of $\Gamma_{o}, \Gamma_{u}$ (calculated with nested sweeps in amplitude and phase) have been traced in the Smith chart corresponding to $\Gamma_{l}$. Although all possible combinations of $\Gamma_{o}, \Gamma_{u}$ have been considered, only results for the worst cases are shown in Fig. 7 to avoid excessive complexity in the representation. In Fig. 7, the amplitudes of the two reflection coefficients are varied between 0.5 and 0.94 and each phase is varied in the range $0^{\circ}$ to $360^{\circ}$. The analysis has been carried out for $P_{\text {in }}=-10 \mathrm{dBm}$, $P_{\text {in }}=10 \mathrm{dBm}$, and $P_{\text {in }}=13 \mathrm{dBm}$. As expected, there is a higher spread of the loci when the circuit behaves more nonlinearly due to the higher influence of $\Gamma_{u}$ on the Hopf locus geometry. The original oscillation is extinguished from certain $P_{\text {in }}$ values (asynchronous extinction) due to the reduction of negative resistance with the excitation amplitude. Indeed, the closest Hopf locus becomes tangent to the unit Smith chart at $P_{\text {in }}=13 \mathrm{dBm}$ and the loci family are out of the unit Smith chart for slightly higher $P_{i n}$.

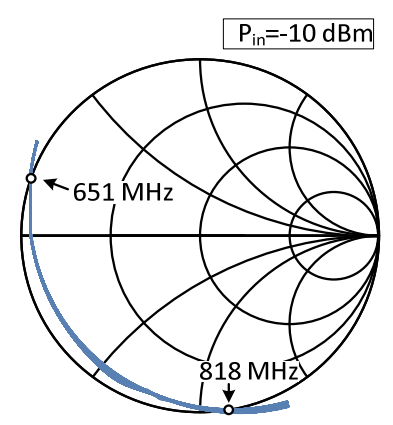

(a)

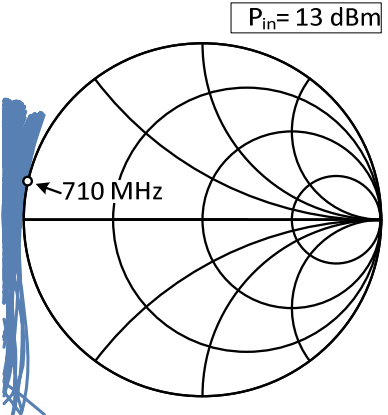

(c)

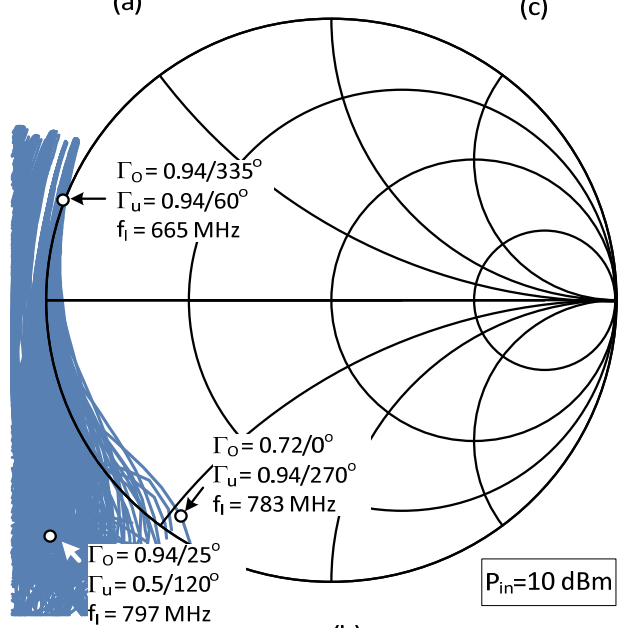

(b)

Fig. 7 Large-signal analysis of the potential instability in the PA of Fig. 1. The Hopf loci obtained for $\left|\Gamma_{o}\right|,\left|\Gamma_{u}\right|$ ranging between 0.5 and 0.94 and phase values going from $0^{\circ}$ to $360^{\circ}$, have been represented for three different input power levels. (a) $P_{\text {in }}=-10 \mathrm{dBm}$. (b) $P_{\text {in }}=10 \mathrm{dBm}$. (c) $P_{\text {in }}=13 \mathrm{dBm}$ (tangency). Some examples of $\left(f, \Gamma_{o}, \Gamma_{u}\right)$ points are shown in the figure.

To rigorously verify the accuracy of the loci, a particular load network exhibiting reflection coefficients within the unstable region for some $P_{\text {in }}$ values will be considered. This load is shown in Fig. 8(a). The particular perturbation frequency $f=19.3 \mathrm{MHz}$ (about the middle of the critical frequency interval) is chosen. The corresponding reflection coefficients $\Gamma_{l}, \Gamma_{o}, \Gamma_{u}$ at the respective frequencies $f_{\text {in }}-f, f_{\text {in }}$ and $f_{\text {in }}+f$ (with $f_{\text {in }}=800 \mathrm{MHz}$ ), are indicated in a table. When keeping $\Gamma_{o}$ and $\Gamma_{u}$ fixed, at each $P_{i n}$ value, there will be a different $\Gamma_{l}$ providing a Hopf bifurcation at the particular frequency $f=19.3 \mathrm{MHz}$. This Hopf bifurcation point will fulfill (16) in the following manner:

$Y_{T}\left(\Gamma_{o}=0.928 e^{-j 149.8^{\circ}}, \Gamma_{u}=0.926 e^{-j 157.1^{\circ}}, f=19.3 \mathrm{MHz}, P_{i n}, \Gamma_{l}\right)=0$.

Of course, once $\Gamma_{o}$ and $\Gamma_{u}$ are fixed, it is unlikely to have a Hopf point exactly at $f=19.3 \mathrm{MHz}, \Gamma_{l}=0.934 \angle-135.6^{\circ}$. In 
order to have the Hopf point exactly at $\Gamma_{l}=0.934 \angle-135.6^{\circ}$, an additional parameter, the gate bias voltage [Fig. 8(b)], has been tuned slightly from $V_{G G}=-2.41 \mathrm{~V}$ to $V_{G G}=-2.167 \mathrm{~V}$. By tuning $V_{G G}$, it has been possible to obtain the Hopf bifurcation at precisely $\Gamma_{l}=0.934 \angle-135.6^{\circ}$ and $f=19.3 \mathrm{MHz}$ for $P_{i n}=-8.71 \mathrm{dBm}$. Fig. $8(\mathrm{~b})$ presents the variation of the Hopf locus points with $P_{\text {in }}$ and $V_{G G}$. As $P_{\text {in }}$ increases, the Hopf bifurcation points move farther away from the center of the unit Smith chart. As expected, these Hopf bifurcation points penetrate less in the unit Smith chart as $V_{G G}$ decreases.

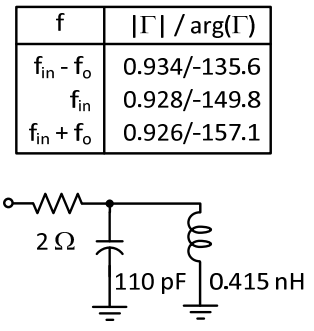

(a)

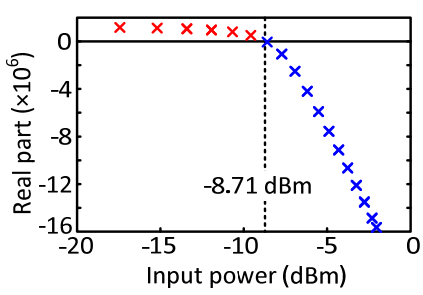

(c)

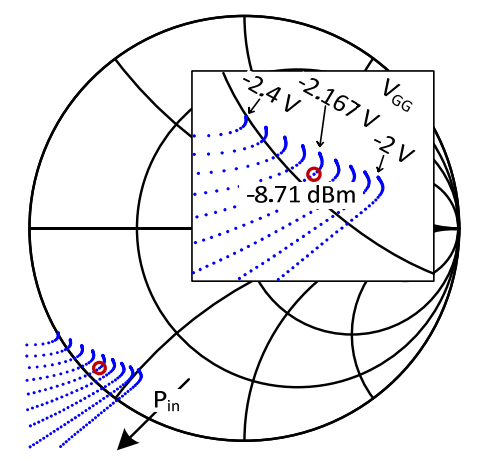

(b)

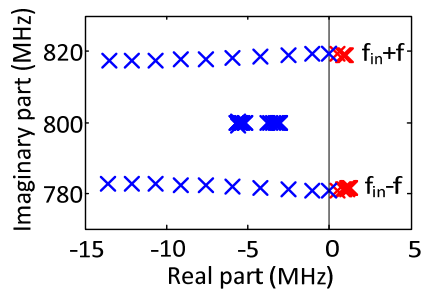

(d)
Fig. 8 Validation of the Hopf bifurcation loci in the nonlinear regime. (a) Load network considered for the analysis of the asynchronous extinction with $f_{\text {in }}=800 \mathrm{MHz}$ and $f=19.3 \mathrm{MHz}$. (b) Hopf bifurcation points at constant $f=19.3 \mathrm{MHz}$, when varying $V_{G G}$ and $P_{i n}$. The Hopf bifurcation point is located exactly at $\Gamma_{l}$ in (a) for $V_{G G}=-2.167 \mathrm{~V}$ and $P_{\text {in }}=-8.71 \mathrm{dBm}$. (c) Pole-zero identification. The real part of the dominant complex conjugate poles is represented versus $P_{i n}$. (d) The two pairs of complex-conjugate poles $f_{\text {in }}-f$ and $f_{\text {in }}+f$ associated with the same Floquet multiplier [11,24] are represented in the complex plane. At the crossing point the lower sideband frequency is $f_{\text {in }}-f=780.7 \mathrm{MHz}$, agreeing fully with the results of the Hopf loci in (b).

From the analysis in Fig. 8(b), with the load network in Fig. 8(a), the circuit should exhibit stable behavior from $P_{\text {in }}=-8.71 \mathrm{dBm}$. This result has been validated (Verification II) with pole-zero identification [21-23,25]. The real part of the dominant pair of complex-conjugate poles has been traced versus the input power in Fig. 8(c). At exactly $-8.71 \mathrm{dBm}$, the pair of complex-conjugate poles crosses from the right side to the left side of the complex plane (Hopf bifurcation). Fig. 8(d) shows the pole evolution in the complex plane. The two pairs of complex-conjugate poles $f_{\text {in }}-f$ and $f_{\text {in }}+f$ (associated with the same Floquet multiplier $[11,24]$ ) cross the imaginary axis at $P_{\text {in }}=-8.71 \mathrm{dBm}$. At the crossing point, the frequency of the poles is $f_{\text {in }}-f=780.7 \mathrm{MHz}$ [Fig. 8(d)], agreeing fully with the prediction of Fig. 8(b). Note that the output harmonic and sideband terminations for $|k| \neq 1$ have little impact on the bifurcation point. Indeed, these terminations were fixed at $Z_{c}$ in the Hopf loci analysis of Fig. 8(b), whereas the analysis of Figs. 8(c) and 8(d) takes into account the actual impedance exhibited by the load network at all harmonic and sideband frequencies. This confirms our original assumption that they would have little relevance due to the output filtering effect.

Measurements of the PA in Fig. 1 have been carried out with a triple-stub tuner. Fig. 9 shows the measurement results. An oscillation was observed in small-signal operation for load values in the critical region of the unit Smith chart. Increasing $P_{\text {in }}$, the oscillation mixed with the input signal [Fig. 9(a)], until eventually vanishing. Fig. 9(a) shows the spectra measured for $P_{\text {in }}=0 \mathrm{dBm}$ and three different positions of the triple-stub tuner. The frequency of the spectral line immediately below the carrier agrees with the lower sideband frequency detected in Figs. 2 and 7. In the three positions considered in Fig. 9(a), the impedance exhibited at the oscillation frequency by the triple-stub tuner belonged to the critical region. The triplestub tuner impedance measured at Position 2 is shown in Fig. 9(b).

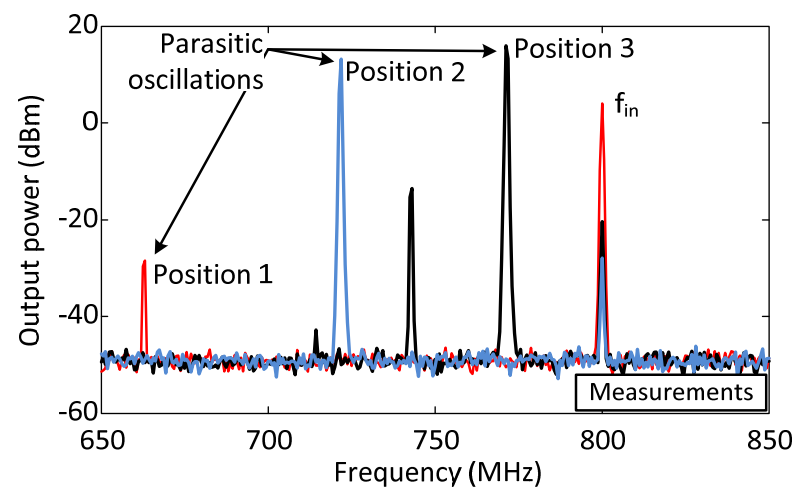

(a)

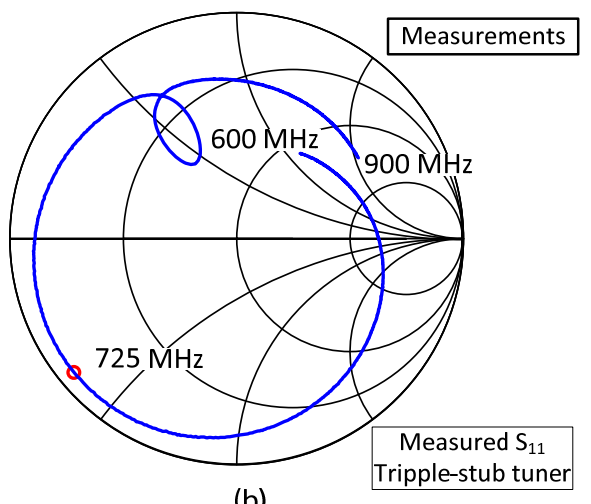

(b)

Fig. 9 Experimental measurements of the amplifier in Fig. 1. (a) Spectra measured for $P_{\text {in }}=0 \mathrm{dBm}$ and three different positions of the triple-stub tuner. (b) Triple-stub tuner $\mathrm{S}_{11}$ measured at Position 2. Measured $S_{11}$ at $725 \mathrm{MHz}$ is marked with a circle (o).

The opposite situation, in which the amplifier is stable in small signal and the oscillation arises from a certain value of 
input power, has also been considered. This phenomenon occurs in the distributed line amplifier of Fig. 10. A low power transistor has been chosen for reasons of nonlinear model availability. This transistor exhibits gain up to about $20 \mathrm{GHz}$, so it should constitute a good test bench for the stability analysis. The parallel capacitance $C_{A}$ (Fig. 10) attenuates spurious passbands of the distributed output network. The Hopf bifurcation loci for the most critical combinations of $\Gamma_{o}$ and $\Gamma_{u}$ values are shown in Fig. 11. They have been obtained through nested sweeps in $\Gamma_{o}$ and $\Gamma_{u}$, covering magnitudes from 0.5 to 0.94 and phases from $0^{\circ}$ to $360^{\circ}$. The input power values considered are $P_{\text {in }}=-22 \mathrm{dBm}$, $P_{i n}=-15 \mathrm{dBm}$ and $P_{\text {in }}=-2 \mathrm{dBm}$. For low $P_{\text {in }}$, the Hopf loci do not enter the unit Smith chart [Fig. 11(a)], but as the input power increases, they approach this chart, suggesting potential instability at higher input power. For $P_{\text {in }}=-15 \mathrm{dBm}$, there is a tangency to the unit Smith chart [Fig. 11(b)] and for $P_{\text {in }}>-15 \mathrm{dBm}$, some of the loci cross the unit Smith chart, indicating the existence of passive load values giving unstable behavior [Fig. 11(c)]. The critical frequency interval can be readily identified by tracing the magnitude $\left|\Gamma_{l}\right|$ versus the perturbation frequency $f$. Fig. 11(d) shows this critical interval with $P_{\text {in }}=-2 \mathrm{dBm}$.

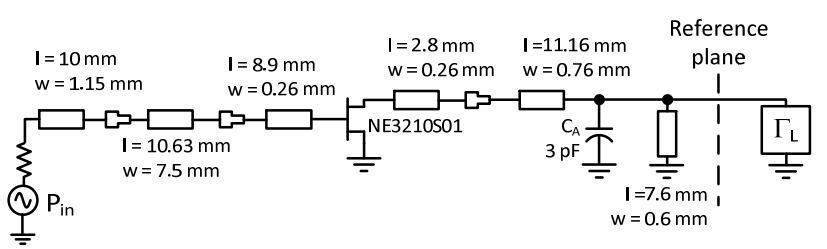

(a)

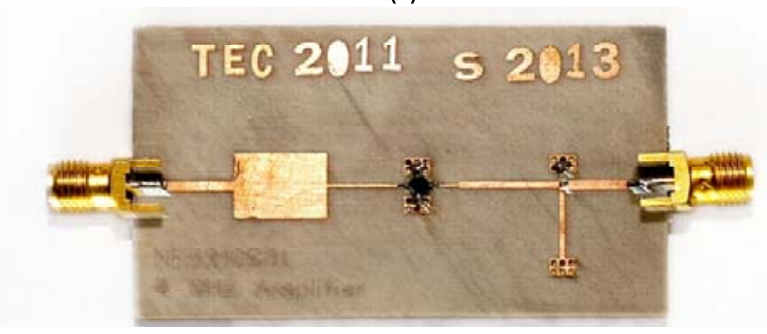

(b)

Fig. 10 (a) Schematic of the distributed line amplifier based on the FET transistor NE3210S01. (b) Photograph of amplifier setup. This amplifier, able to provide $11 \mathrm{~dB}$ gain at $4 \mathrm{GHz}$ for $V_{G G}=-0.25 \mathrm{~V}$, will be used here as a stability test bench, with $V_{G G}=-1.25 \mathrm{~V}$, to obtain gain expansion with input power. The transistor is biased using ZX85-12G-S+ (Mini-Circuits) bias-tees.

For an additional validation, two different load networks, "a" and "b", have been considered. For each load ("a" or "b"), $\Gamma_{L}\left(f_{\text {in }}\right)$, together with the whole set of values taken by the reflection coefficient $\Gamma_{L}\left(f_{\text {in }}+f\right)$, with $0<f<f_{\text {in }} / 2$, are calculated separately from the circuit. For each load "a" and "b", the reflection coefficient at $f_{i n}$ is fixed at the corresponding value $\Gamma_{o} \equiv \Gamma_{L}\left(f_{i n}\right)$. Then, the reflection coefficient at the upper sideband, $\Gamma_{u}$, is swept covering the whole set of values of $\Gamma_{u} \equiv \Gamma_{L}\left(f_{\text {in }}+f\right)$ (in magnitude and phase) obtained from the previous separate analysis of the particular load. A Hopf locus is traced for each $\Gamma_{u}$. In this particular example, the first load "a" corresponds to a resistor $(R=120 \Omega)$ and a capacitor $(C=1.906 \mathrm{pF})$ connected in parallel. The input power was varied up to $10 \mathrm{dBm}$ [Fig. 12(a)]. The corresponding reflection coefficient $\Gamma_{L}\left(f_{\text {in }}-f\right)$, evaluated in the maximum critical frequency interval, has been represented in the Smith chart (referred to as "a"). It does not enter the "unstable" region. The second load "b" corresponds to a similar parallel $R C$ load, with a different resistor value: $\mathrm{R}=500 \Omega$. The corresponding reflection coefficient $\Gamma_{L}\left(f_{\text {in }}-f\right)$, evaluated in the maximum critical frequency interval, (referred to as "b") lies in the unstable region [Fig. 12(b)].

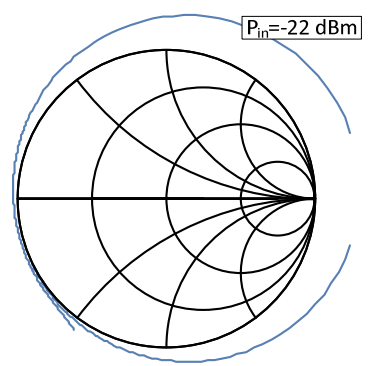

(a)

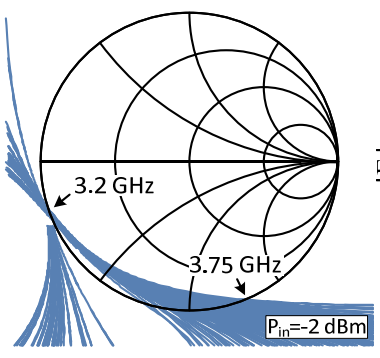

(c)

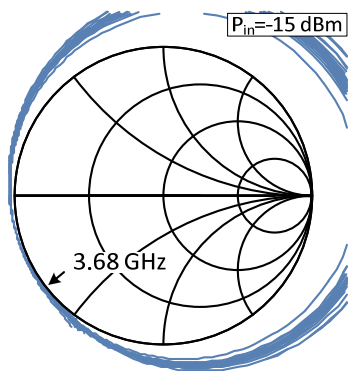

(b)

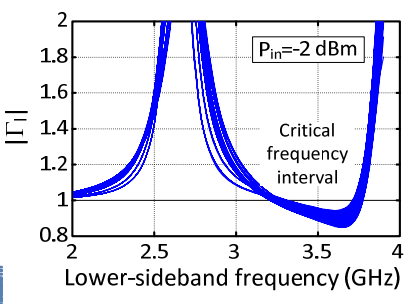

(d)
Fig. 11 Hopf loci for the amplifier in Fig. 10. (a), (b) and (c) Hopf loci obtained for $\left|\Gamma_{o}\right|,\left|\Gamma_{u}\right|$ ranging between 0.5 and 0.94 , and phases going from $0^{\circ}$ to $360^{\circ}$, at three different input power levels: (a) $P_{\text {in }}=-22 \mathrm{dBm}$ (stable behavior). (b) $P_{\text {in }}=-15 \mathrm{dBm}$ (tangency). (c) $P_{i n}=-2 \mathrm{dBm}$ (potential instability). (d) Frequency variation of the magnitude of the lower sideband reflection coefficient $\left|\Gamma_{l}\right|$ at the Hopf locus for $P_{\text {in }}=-2 \mathrm{dBm}$.

The results obtained with the Hopf bifurcation loci have been validated (Verification III) with pole-zero identification [21-23,25]. In the case of the first load network with frequency variation "a", the poles of the periodic solution calculated versus the input power [Fig. 13(a)], never cross the imaginary axis to the right-hand side of the complex plane, indicating stable behavior. Note that the frequency of the dominant poles is contained within the critical frequency interval detected in Fig. 11(d). In the case of the second load network with frequency variation " $b$ ", the poles of the periodic solution calculated versus the input power [Fig. 13(b)], cross to the right-hand side of the complex plane at the input power $P_{\text {in }}=-10 \mathrm{dBm}$, confirming the unstable behavior. The same pair of poles crosses again to the left-hand side at $P_{i n}=7.5 \mathrm{dBm}$. At each of these two power values, one of the 
loci intersects with the load characteristic $\Gamma_{L}\left(f_{\text {in }}-f\right)$. In the $P_{\text {in }}$ interval $-10 \mathrm{dBm}$ to $7.5 \mathrm{dBm}$, the frequency of the critical poles in Fig. 13(b) is contained in the dangerous frequency interval detected in Fig. 11(d).

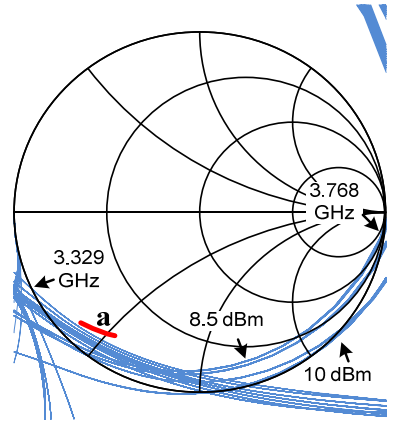

(a)

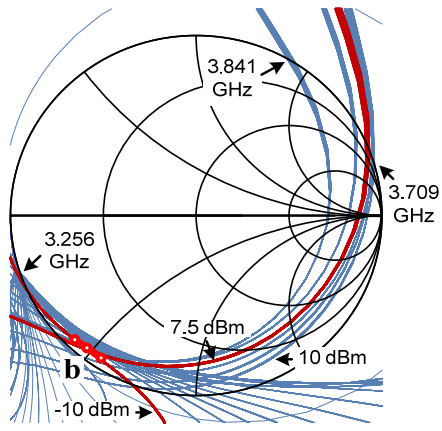

(b)
Fig. 12 Validation of the large-signal stability analysis, using two load networks. Input power variations in the interval $P_{\text {in }}=-22 \mathrm{dBm}$

to $10 \mathrm{dBm}$ are considered. (a) Parallel connection of $R=120 \Omega$, $C=1.906 \mathrm{pF}$. In the critical frequency interval, it gives rise to the curve "a". (b) Parallel connection of $R=500 \Omega, C=1.906 \mathrm{pF}$. It gives rise to the curve "b".

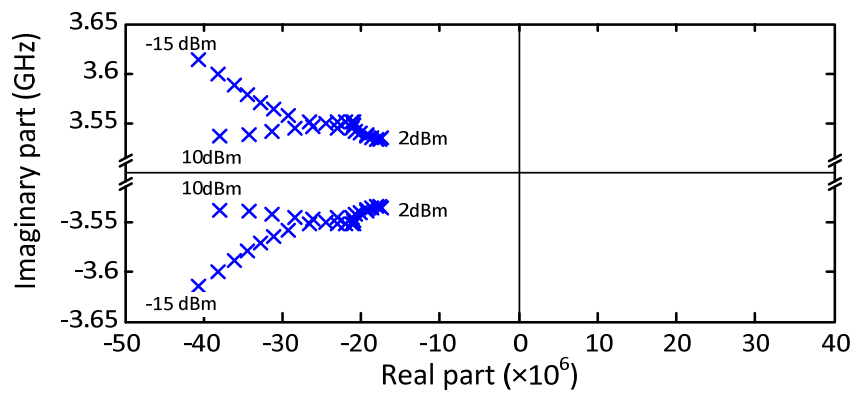

(a)

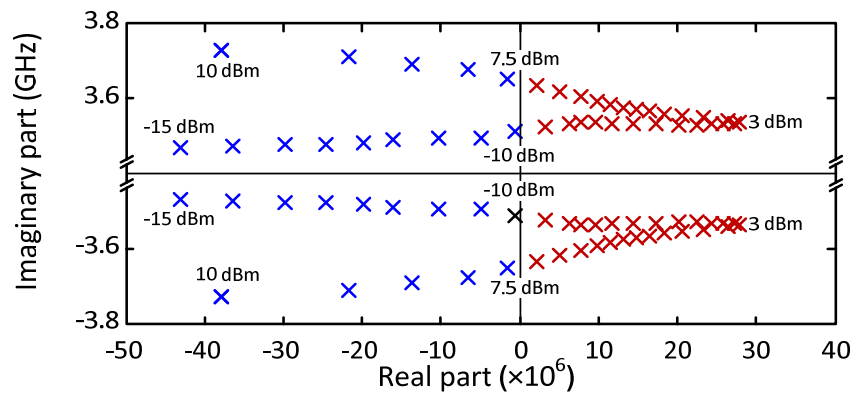

(b)

Fig. 13 Validation with pole-zero identification of the results of the analysis based on the Hopf bifurcation loci in Fig. 12. (a) Variation of the circuit's dominant poles with the input power in the interval $P_{\text {in }}=-22 \mathrm{dBm}$ to $10 \mathrm{dBm}$ when the circuit is loaded with "a" in Fig. 12. (b) Variation of the circuit's dominant poles with the input power when the circuit is loaded with "b" in Fig. 12. The complexconjugate poles cross to the right-hand side of the complex plane at $P_{\text {in }}=-10 \mathrm{dBm}$ and to the left-hand side of this plane at $P_{\text {in }}=7.5 \mathrm{dBm}$.
Note that in some cases, the mismatch effects may also affect the behavior about the frequencies $k f_{\text {in }}$ of the steadystate solution. In that case, the arrangement in Fig. 6 to calculate the loci would remain valid, although more load terminations implemented with the aid of ideal filters should be considered. For each set of termination impedances, a Hopf locus would be obtained using the small-signal current source in an identical manner. Thus, more nested sweeps should be carried out to obtain the loci. In order to reduce the number of sweeps, it will be possible to derive general conditions for potential instability, which will be shown in the next section.

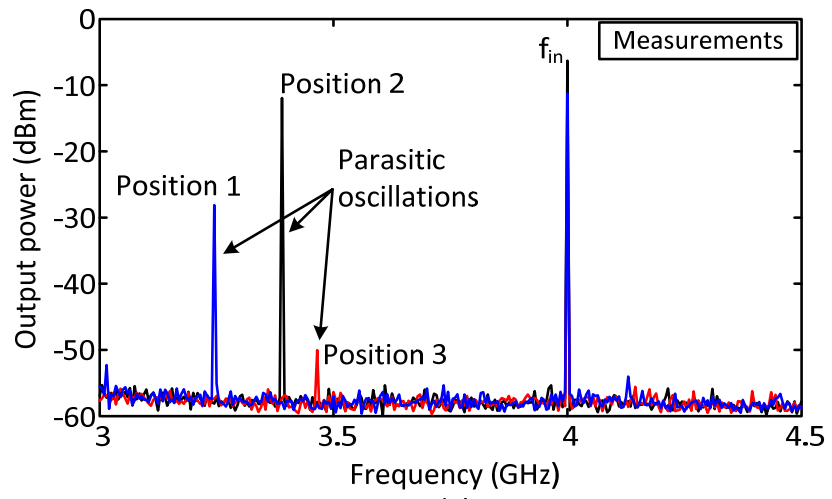

(a)

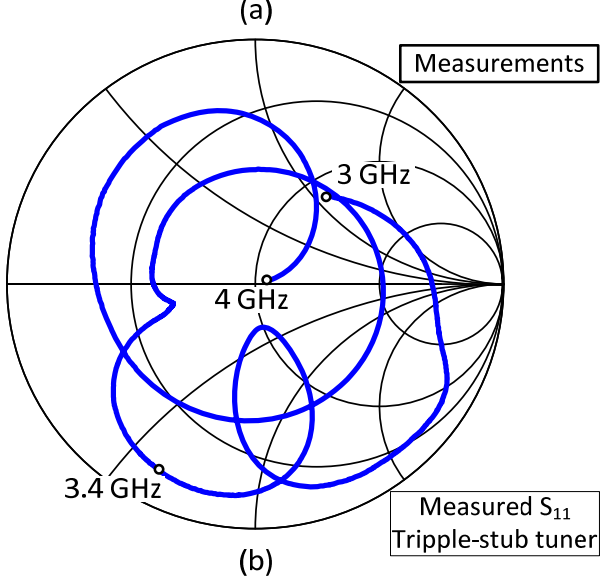

Fig. 14 Experimental measurements of the amplifier in Fig. 10. (a) Spectra measured for $P_{\text {in }}=0 \mathrm{dBm}$ and three different positions of the triple-stub tuner. (b) Triple-stub tuner $\mathrm{S}_{11}$ measured at Position 2.

The amplifier in Fig. 10 has also been experimentally characterized. Fig. 14 shows the measurement results. With the original $50 \Omega$ termination, it is stable for all input power values. Using a triple-stub tuner, it was impossible to lead the amplifier to instability below the input power value $P_{\text {in }}=-5$ $\mathrm{dBm}$. Above this power, for some positions of the triple-stub tuner, a self-oscillation mixed with the drive signal. Fig. 14(a) shows the spectra measured for $P_{i n}=0 \mathrm{dBm}$ and three different positions of the tuner. The frequency of the spectral line immediately below the carrier agrees with the lower sideband frequency detected in Fig. 11(d). Note that this oscillation frequency belongs in all cases to the critical 
interval $(3.2 \mathrm{GHz}, 3.7 \mathrm{GHz})$, in agreement with the simulation results. In the three positions considered in Fig. 14(a), the impedance exhibited at the oscillation frequency by the triplestub tuner belongs to the critical region. The impedance measured at Position 2 is shown in Fig. 14(b). The difference between the minimum input power required to lead the amplifier to instability in simulation and measurements is attributed to loss in the triple-stub tuner, preventing this tuner from exhibiting impedances close to the boundary of the unit Smith chart.

\section{Potential INSTABILITy ANALYSIS UNDER MismatCH EFFECTS}

Defining a "virtual" scattering matrix with the circuit output as a single reference plane, it will be possible to derive absolute stability criteria versus mismatch effects at two sidebands, for instance $f_{l}$ and $f_{u}$, located about $f_{\text {in }}$, or at $f$ and $f_{\text {in }}-f$. The criteria should be evaluated for all possible values of the reflection coefficient at the input frequency $\Gamma_{o}$. If terminations at other harmonic and sideband frequencies are also relevant, they can be taken into account, verifying the absolute stability criteria for all possible combinations of their corresponding reflection coefficients by means of the nested sweep technique. These absolute stability conditions are presented in the following.

Using a derivation identical to the one from (9) to (12), it is possible to obtain an outer-tier system in the two sideband frequencies of interest $f_{l}$ and $f_{u}$ :

$$
\begin{aligned}
& \Delta V_{l}^{*}=Z_{l, l} \Delta I_{l}^{*}+Z_{l, u} \Delta I_{u} \\
& \Delta V_{u}=Z_{u, l} \Delta I_{l}^{*}+Z_{u, u} \Delta I_{u}
\end{aligned}
$$

where the same notation given in (12) has been used. For analysis of the absolute stability conditions, the impedance matrix in (17) must indeed be calculated in commercial software, unlike the matrix in (11) that was only used for explanatory purposes. With an impedance matrix (instead of an admittance one) current excitations and voltage state variables can be used, as in the analysis (9)-(16).

The impedance matrix in (17) is calculated in a very simple manner using small-signal / large-signal analysis in commercial HB software. A sketch of the two circuits used to obtain the four components of the impedance matrix is shown in Fig. 15. Ideal filters and ideal loads are connected to the circuit output to ensure the required terminations. The fundamental frequency is loaded with $\Gamma_{o}$. Other harmonic frequencies $k f_{\text {in }}$ (with $|k| \neq 1$ ), as well as the non-critical sidebands $k f_{\text {in }}+f$ (also with $|k| \neq 1$ ) are loaded with the default value $\Gamma=0$. The two dominant sideband frequencies $f_{l}$ and $f_{u}$ are in open circuit termination (or loaded with very high impedance). Note that any impedance variation at the sideband frequencies should not affect the steady-state solution, which is inherently ensured by the analysis through the conversion matrix approach.
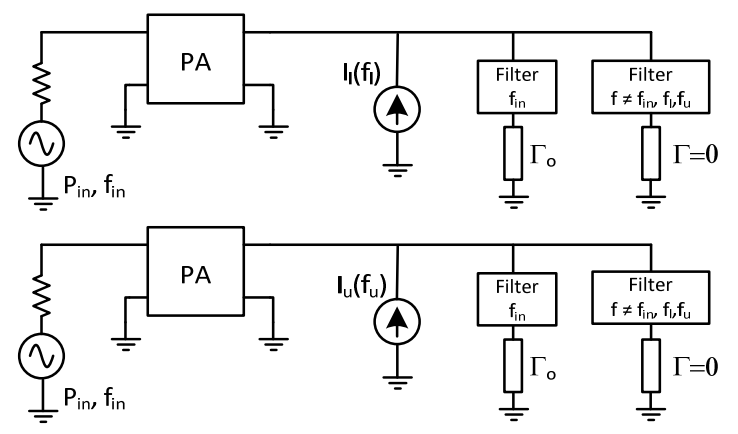

Fig. 15 Sketch of the two subcircuits used for the calculation of the four terms of the $2 \times 2$ impedance matrix in (17).

In general, commercial HB software will only permit the introduction of a current source at the sidebands $f_{\text {in }}+f$ or $f_{\text {in }}-f$, that is, only at positive multiples of $f_{\text {in }}$. To determine $Z_{l, l}$ and $Z_{u, l}$, a current source $I_{l}$, operating at the lower frequency sideband $f_{l}=f_{\text {in }}-f$, is introduced into the circuit [Fig. 15(a)]. When the upper sideband $f_{u}=f_{\text {in }}+f$ is under open-circuit conditions, the two parameters $Z_{l, l}$ and $Z_{u, l}$ are calculated as:

$$
\begin{aligned}
& Z_{l, l}=\frac{\Delta V_{l}{ }^{*}}{I_{l}{ }^{*}}=\operatorname{conj}\left[\frac{V\left(f_{\text {in }}-f\right)}{I\left(f_{\text {in }}-f\right)}\right] \\
& Z_{u, l}=\frac{\Delta V_{u}}{I_{l}{ }^{*}}=\frac{V\left(f_{\text {in }}+f\right)}{I\left(-f_{\text {in }}+f\right)}
\end{aligned}
$$

To determine $Z_{l, u}$ and $Z_{u, u}$, a current source $I_{u}$, operating at the upper frequency sideband $f_{u}=f_{\text {in }}+f$, is introduced into the circuit [Fig. 15(b)]. When the lower sideband $f_{l}=f_{\text {in }}-f$ is under open-circuit conditions, the two parameters $Z_{l, u}$ and $Z_{u, u}$ are obtained as:

$$
Z_{l, u}=\frac{\Delta V_{l}^{*}}{I_{u}}=\frac{V\left(-f_{\text {in }}+f\right)}{I\left(f_{\text {in }}+f\right)}, \quad Z_{u, u}=\frac{\Delta V_{u}}{I_{u}}=\frac{V\left(f_{\text {in }}+f\right)}{I\left(f_{\text {in }}+f\right)}
$$

In the criteria defined here, a particular steady-state solution will be potentially unstable under mismatch effects when for some passive termination $Z_{L, u}$ at the upper sideband $f_{u}$, it exhibits negative resistance at the lower sideband $f_{l}$, that is, $\operatorname{Re}\left(Z_{\text {out }, l}\right)<0$, or, reciprocally, when for some passive termination $Z_{L, l}$ at the lower sideband $f_{l}$, it exhibits negative resistance at the upper sideband $f_{u}$, that is, $\operatorname{Re}\left(Z_{\text {out }, u}\right)<0$. For the analysis of potential instability, the perturbation frequency $f$ must be swept in the interval $\left(0, f_{\text {in }} / 2\right)$, combining the data resulting from the simulation of the two circuits: with the current source $I_{l}$ and with the current source $I_{u}$.

The definition of potential instability under large-signal behavior is formally identical to Rollet's definition for potential instability in small signal. However, there are essential differences. Rollet's definition refers to the two ports of a linear network, linearized about the $d c$ solution. The only frequency existing in the analysis is the perturbation frequency $f$. The description in (17) refers to only one physical port: the output port connected to the antenna or the next block in the 
system chain. For each $\Gamma_{o}$ (load at the fundamental frequency $f_{\text {in }}$ ), the circuit is linearized from its output terminals using the small-signal / large-signal analysis in HB software. This analysis is used to accurately calculate the $2 \times 2$ matrix that linearly relates currents and voltages at the two sideband frequencies $f_{l}=f_{\text {in }}-f$ and $f_{u}=f_{\text {in }}+f$. Note that there is no approximation in this calculation since all the harmonic terms and sidebands, for $|k| \neq 1$, are implicitly taken into account in the HB and conversion matrix analyses in Fig. 15.

As discussed above, the current and voltages at the two different sidebands $f_{l}$ and $f_{u}$ can be seen as currents and voltages at the two ports of a virtual two-port network. Thus, it will be possible to perform a matrix transformation, from the impedance matrix in (17) to a virtual scattering matrix using conventional transformation formulae [38]:

$$
\left[\begin{array}{ll}
Z_{l, l} & Z_{l, u} \\
Z_{u, l} & Z_{u, u}
\end{array}\right] \rightarrow\left[\begin{array}{ll}
S_{l, l} & S_{l, u} \\
S_{u, l} & S_{u, u}
\end{array}\right]
$$

Thus, conditional or unconditional stability can be determined using standard procedures, that is, calculating both the " $k$ " factor and determinant of the scattering matrix or the " $\mu$ " factor [39]. In large-signal operation, the absolute stability conditions versus mismatch effects are given by $[27,31,38]$ :

$$
\begin{aligned}
& k_{L S}(f)=\frac{1-\left|S_{l l}\right|^{2}-\left|S_{u u}\right|^{2}+\Delta_{L S}^{2}}{2\left|S_{l u} S_{u l}\right|}>1 \\
& \left|\Delta_{L S}(f)\right|=\left|S_{l l} S_{u u}-S_{l u} S_{u l}\right|<1
\end{aligned}
$$

where $\Delta_{L S}$ is the determinant of the scattering type matrix. The above conditions must be fulfilled for all the perturbation frequencies $f$ in the interval $\left(0, f_{\text {in }} / 2\right)$. Note that a single condition can only be used in terms of the $\mu$ factor [39]. The computation of conditions (21) is directly carried out by commercial software when the new scattering type matrix, calculated with the two circuits in Fig. 15, is provided as input.

Conditions (21) will indicate absolute stability provided the nonlinear circuit is stable on its own, which can be verified with a proviso referred to the two dominant sidebands $f_{\text {in }}-f$ and $f_{\text {in }}+f$. When loading the two sidebands with infinite and zero impedances, the circuit must be stable for all $P_{\text {in }}$ and $\Gamma_{o}$ values. The harmonic components and the sidebands $k f_{\text {in }}+f$, with $|k| \neq 1$ are loaded with the original load $Z_{c}=50 \Omega$ in this analysis. If the proviso is satisfied, instability will be due to the particular load values at the sidebands frequencies $f_{\text {in }}-f$ and $f_{\text {in }}+f$. The circuit will be conditionally stable if the inequalities (21) are not fulfilled. In the case of more relevant sidebands, it would be possible to adapt multiport generalizations of Rollet's conditions [28-29].

The new theory has been validated through application to the amplifier in Fig. 10 biased at the point $V_{G G}=-1.25 \mathrm{~V}$, $V_{D D}=3 \mathrm{~V}$. For simplicity, the termination impedance at $f_{\text {in }}$ is considered to be $\Gamma_{o}=0$. This can correspond to practical situations in which it is possible to implement extra matching at the drive frequency. The variation of $k_{L S}$ and $\left|\Delta_{L S}\right|$ versus the perturbation frequency $f$ for increasing values of input power $P_{\text {in }}$ is shown in Fig. 16. Note that for each input power, a different steady-state is calculated about which the circuit is linearized with the conversion matrix approach. As can be seen in Fig. 16(a), the circuit is unconditionally stable under small signal $\left(P_{i n}=-22 \mathrm{dBm}\right)$, since $k_{L S}$ is always greater than 1 and $\left|\Delta_{L S}\right|<1$. When the input power increases, it becomes conditionally stable at the input power $P_{i n}=-14.31 \mathrm{dBm}$. This power is larger than the one in the analysis of Fig. 11, since in this case, the fundamental frequency is loaded with $\Gamma_{o}=0$, whereas in Fig. 11 a double sweep in $\Gamma_{o}$ and $\Gamma_{u}$ has been performed (considering all the possible values of $\Gamma_{o}$ ). At this power value, $k_{L S}$ is tangential to 1 [Fig. 16(b)]. For larger $P_{\text {in }}$ values, $k_{L S}$ is always less than one in a certain interval of the perturbation frequency [Fig. 16(c)]. Of course, if variations in $\Gamma_{o}$ were considered, at each input power one would have to trace the $k_{L S}$ and $\left|\Delta_{L S}\right|$ curves corresponding to each $\Gamma_{o}$. This is because the steady-state changes with each $\Gamma_{o}$ and so does the virtual scattering matrix calculated with the conversion matrix approach.

For validation, the results of the analysis based on $k_{L S}$ and $\left|\Delta_{L S}\right|$ have been compared with those obtained through the nested sweep of reflection coefficient values (technique used in Section III). Because in the analysis of (21) the reflection coefficient at $f_{\text {in }}$ is $\Gamma_{o}=0$, only sweeps in the amplitude and phase of $\Gamma_{u}$ have been carried out at each $P_{i n}$ value. The results are shown in the Smith charts on the right side (Fig. 16). For $P_{i n}=-14.31 \mathrm{dBm}$, one of the Hopf loci becomes tangent to the Smith chart, in agreement with the $k_{L S}$ tangency to 1 in Fig. 16(b). For higher power, the critical frequency interval agrees with the one detected with the nested sweeps. In conclusion, the results obtained with the independent simulations through reflection coefficient sweeps validate the potential instability predictions with $k_{L S}$ and $\left|\Delta_{L S}\right|$ (Verification IV).

The particular passive load corresponding to the tangency in Fig. 16(b) has been determined with the $\Gamma_{u}$ sweep and from inspection of the $\Gamma_{l}$ Smith chart. The frequency of this tangency is $f_{l}=3.707 \mathrm{GHz}$ and the tangent load is $\Gamma_{l}=0.99 /-132^{\circ}, \Gamma_{o}=0$ and $\Gamma_{u}=0.99 /-17^{\circ}$. A network exhibiting approximately these reflection coefficient values (at $f_{i n}, f_{l}$ and $f_{u}$ ) has been synthesized with LCR elements (Fig. 17). With such values, the circuit should exhibit a Hopf bifurcation at $P_{\text {in }}=-14.31 \mathrm{dBm}$ and $f_{l}=3.707 \mathrm{GHz}$. Moreover, due to the tangency of $k_{L S}$, this must be the only output load for which the circuit is able to oscillate at $P_{\text {in }}=-14.31 \mathrm{dBm}$, and the oscillation frequency at this tangency should be $f_{l}=3.707 \mathrm{GHz}$. This has been validated by tracing the Hopf bifurcation locus of the circuit loaded with the network shown in Fig. 17 using an independent procedure [11] (Verification $V$ ). This procedure is based on the use of an $\mathrm{AG}$ with small amplitude $\mathrm{A}_{\mathrm{AG}}=\varepsilon$ (oscillation boundary 
condition [11]) to detect the Hopf bifurcations of the amplifier circuit terminated with the load network in Fig. 17. Keeping $C_{1}$ constant, the capacitance $C_{2}$, together with the input power $P_{\text {in }}$, are taken as analysis parameters. These two parameters define a plane (Fig. 18) and the Hopf locus traced in this plane will constitute the boundary between oscillatory and nonoscillatory regions in the circuit terminated with the load network in Fig. 17.
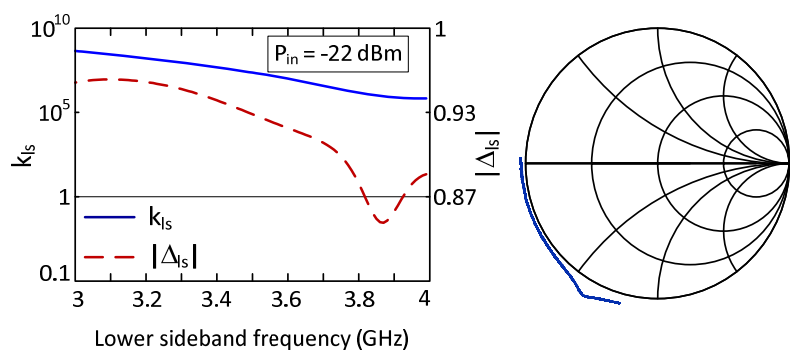

(a)

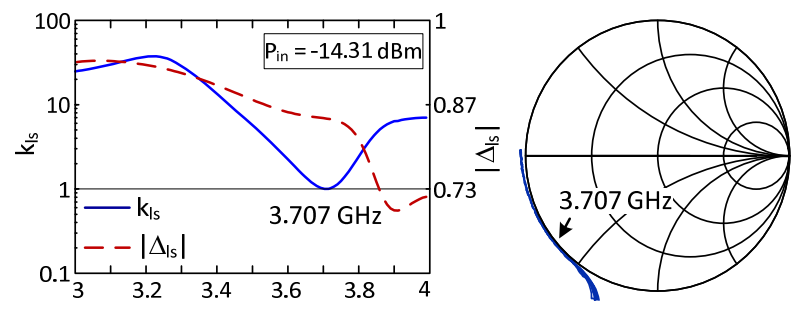

Lower sideband frequency $(\mathrm{GHz})$

(b)

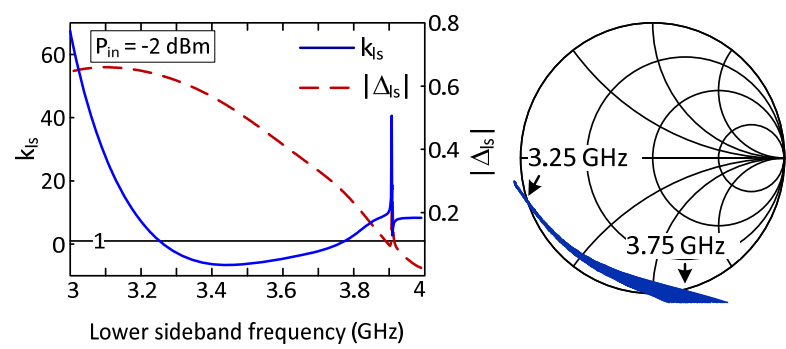

(c)

Fig. 16 Analysis of unconditional stability applied to the PA in Fig. 10 for three different values of input power. (a) $P_{\text {in }}=-22 \mathrm{dBm}$. (b) $P_{\text {in }}=-14.31 \mathrm{dBm}$. (c) $P_{\text {in }}=-2 \mathrm{dBm}$.

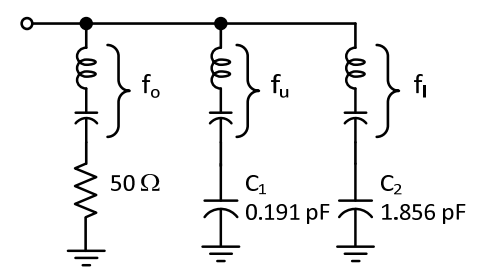

\begin{tabular}{|r|r|}
\hline$f[G H z]$ & \multicolumn{1}{|c|}{$\Gamma$} \\
\hline 3.707 & $0.999 /-132.1^{\circ}$ \\
4 & $0.011 / 90.6^{\circ}$ \\
4.293 & $0.990 /-17.1^{\circ}$ \\
\hline
\end{tabular}

Fig. 17 Output load fulfilling the tangency condition in Fig. 16(b). This load is connected to the output (reference) plane, replacing the ideal terminations. The reflection coefficient values exhibited by this load at the perturbation frequency $(3.707 \mathrm{GHz})$ corresponding to the tangency are shown in the table. Due to implementation inaccuracies, the reflection coefficient values are slightly different from those at the tangency $\left(\Gamma_{l}=0.99 /-132^{\circ}, \Gamma_{o}=0\right.$ and $\left.\Gamma_{u}=0.99 /-17^{\circ}\right)$.

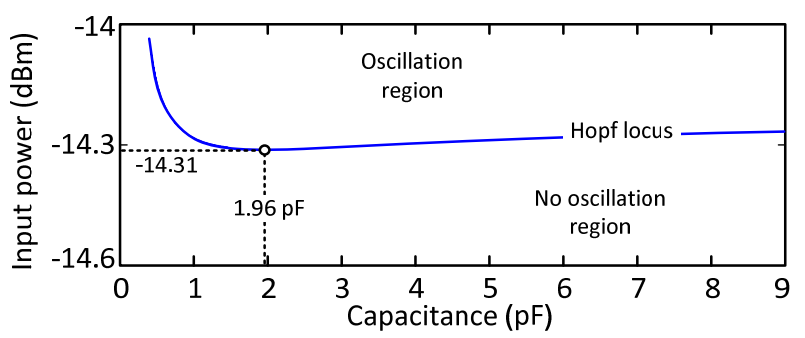

(a)

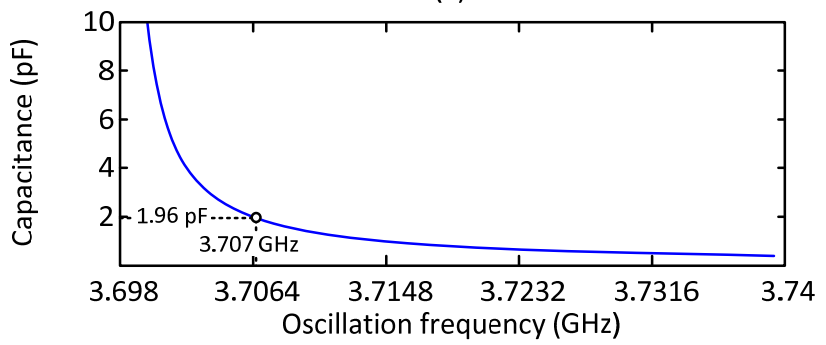

(b)

Fig. 18 Validation of the tangency condition in Fig. 16(b) by obtaining the Hopf bifurcation locus of the circuit terminated with the load network of Fig. 17. (a) Hopf bifurcation locus in the plane defined by $C_{2}$ and $P_{i n}$. The straight line $P_{\text {in }}=-14.31 \mathrm{dBm}$ is tangent to the Hopf locus at $\mathrm{C}_{2}=1.96 \mathrm{pF}$, corresponding to the oscillation frequency $f_{l}=3.707 \mathrm{GHz}$. (b) Capacitance $C_{2}$ traced versus $f l$. This representation shows that the tangency occurs at the perturbation frequency predicted in Fig. 16(b).

To obtain the Hopf locus in the plane $P_{i n}, C_{2}$, the AG with very small amplitude $\mathrm{A}_{\mathrm{AG}}=\varepsilon$ at the frequency $f_{\mathrm{AG}}=f_{l}$ is connected to the transistor drain node [11]. Then the Hopf locus is defined by the complex equation $Y_{A G}\left(P_{i n}, C_{2}, f_{l}\right)=0$, where $Y_{A G}$ is the current-to-voltage ratio of the small-signal AG. To trace the locus, the oscillation frequency $f_{l}$ has been swept, optimizing $P_{\text {in }}$ and $C_{2}$ at each sweep step so as to fulfill the non-perturbation condition $Y_{A G}\left(P_{i n}, C_{2}\right)=0$ with the conversion matrix approach [11]. This is more demanding than the calculation of the Hopf loci versus the output load (in Figs. 7 and 11, for instance). Those are obtained through a simple evaluation of a small-signal admittance function [see (1) and (16)] and do not require any optimization.

As deduced from Fig. 18, the Hopf locus is tangent to the line $P_{\text {in }}=-14.31 \mathrm{dBm}$ at $C_{2}=1.9 \mathrm{pF}$, indicating that no oscillation is possible below this power value for any $C_{2}$, in full consistency with the tangency condition of Fig 16(b). We believe that the agreement with this totally independent simulation, taking into account the full frequency variation of the load (at harmonic and sideband frequencies) strongly validates the accuracy of the method.

Next, the influence of load impedances at harmonic components $k f_{\text {in }}$ and sidebands $k f_{\text {in }}+f$, with $|k| \neq 1$, on $k_{L S}$ and $\left|\Delta_{L S}\right|$ will be analyzed. In view of the previous results, their impact must be low. In Fig, 19, the evaluation of $k_{L S}$ and $\left|\Delta_{L S}\right|$ versus frequency has been performed for three different 
values of the termination impedances at harmonic and sideband frequencies with $|k| \neq 1$. As can be seen, only small variations are noticed. The critical frequency interval is the same in the three cases and so is the minimum value of $k_{L S}$. The $k_{L S}$ and $\left|\Delta_{L S}\right|$ values for the three different loading impedances at $|k| \neq 1$ are nearly identical, with small discrepancies for very large $k_{L S}$ values. As another example, Fig. 20 shows the same analysis for the power amplifier in Fig. 1 at the input power $P_{\text {in }}=-0.45 \mathrm{dBm}$. The factor $k_{L S}$ is near tangent to one since this is the power value from which the amplifier stabilizes with $\Gamma_{o}=0$. The agreement for the three different termination impedances is better with the amplifier in Fig. 1 due to a stronger filtering action in the output network of this amplifier.

Characterization of antenna mismatch may provide a maximum VSWR, such that the antenna will never exceed certain $\rho_{\max }$ under mismatch effects [1-8]. If this value can be estimated, the two factors $\mu$ and $\mu$ ' [39] (instead of $k_{L S}$ and $\left.\Delta_{L S}\right)$ may be especially useful to predict the possible instability under a particular $\rho_{\max }$. This is because at each perturbation frequency $f$, they will provide the minimum magnitudes of the sideband reflection coefficients $\left(\left|\Gamma_{l}\right|\right.$ and $\left.\left|\Gamma_{u}\right|\right)$ from which negative resistance (at the opposite sideband) will be obtained. No examples are shown of these analysis since the possible use of the large-signal factors $\left(\mu\right.$ and $\left.\mu^{\prime}\right)$ is immediate once the validity of $k_{L S}$ and $\Delta_{L S}$ has been demonstrated.

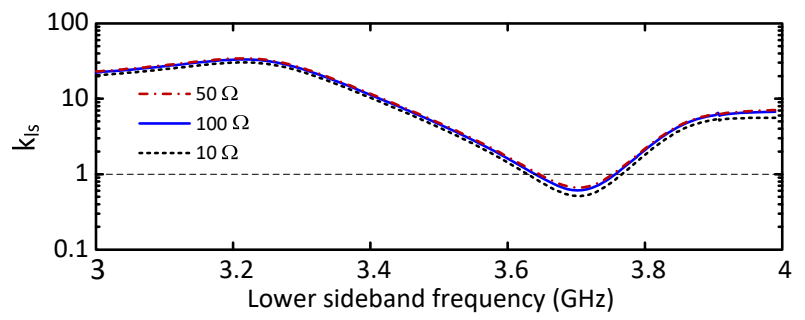

(a)

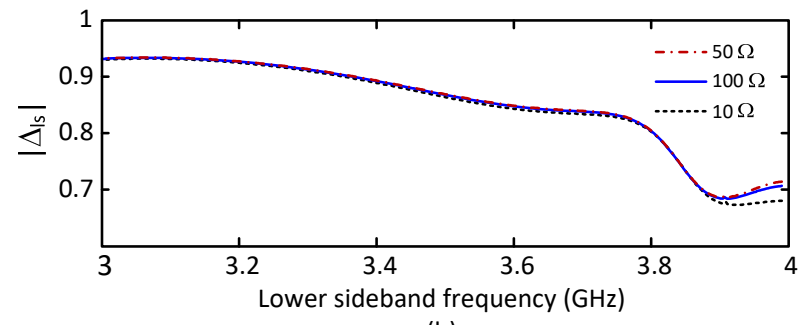

(b)

Fig. 19 Amplifier in Fig. 10, with input power $P_{\text {in }}=-14 \mathrm{dBm}$. Evaluation of $k_{L S}$ and $\left|\Delta_{L S}\right|$ versus the lower sideband frequency has been performed for three different values of the termination impedances at harmonic and sideband frequencies with $|k| \neq 1$.

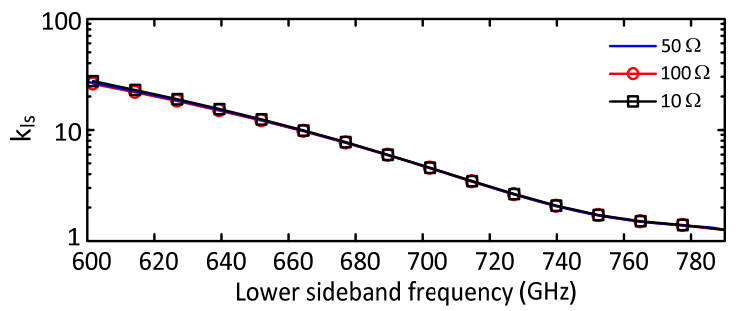

(a)

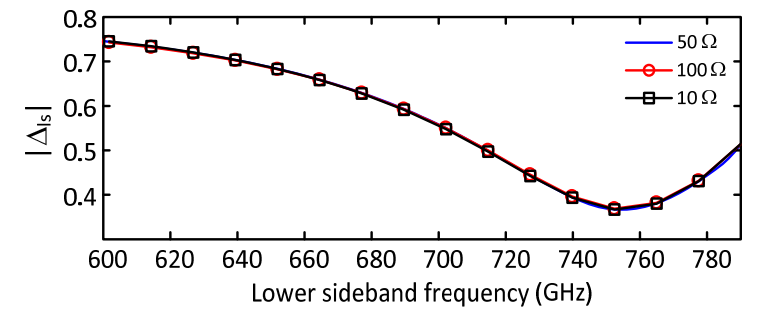

(b)

Fig 20 Amplifier in Fig. 1. Evaluation of $k_{L S}$ and $\left|\Delta_{L S}\right|$ versus the lower sideband frequency has been performed for three different values of the termination impedances at harmonic and sideband frequencies with $|k| \neq 1$.

Tracing the stability circles (with the same geometrical meaning as Rollet's stability circles), one can determine the impedance values at $f_{l}=f_{\text {in }}-f\left(f_{u}=f_{\text {in }}+f\right)$ that give rise to negative resistance when looking into the circuit output at the other sideband $f_{u}\left(f_{l}\right)$. The expressions for the center and radius of these circles are identical to those in Rollet theory, with subindex $l$ being analogous to subindex 1 in that theory and subindex $u$ being analogous to subindex 2 .

Let a fixed perturbation frequency $f$ be considered. For each input power $\left(P_{i n}\right)$ and each $\Gamma_{o}$ value, a stability circle in the $\Gamma_{u}$ plane will delimit the $\Gamma_{u}$ values providing negative resistance at $f_{l}=f_{\text {in }}-f$. To obtain the Hopf loci points in the $\Gamma_{l}$ plane, a series of $\Gamma_{u}$ circles or portions of circles, covering the whole "unstable" region in the $\Gamma_{u}$ plane, will be considered. For each point $p_{u}$ of each $\Gamma_{u}$ circle (at the perturbation frequency $f$ ), the input reflection coefficient (looking into the circuit output at $f_{l}=f_{\text {in }}-f$ ) is calculated through:

$$
\Gamma_{i n, l}\left(p_{u}\right)=S_{l, l}+\frac{S_{l, u} S_{u, l} p_{u}}{1-S_{u, u} p_{u}}
$$

The corresponding point of the Hopf locus in the $\Gamma_{l}$ plane is given by:

$$
Y_{L, l}=-Y_{o u t, l}\left(p_{u}\right)=-Y_{c} \frac{1-\Gamma_{i n, l}\left(p_{u}\right)}{1+\Gamma_{i n, l}\left(p_{u}\right)}
$$

Then, for each $\Gamma_{u}$ circle (or portion of circle) in the unstable region, one obtains a circle (or portion of circle) of Hopf loci points (at the particular perturbation frequency $f$ ) in the $\Gamma_{l}$ plane, defined by the following equation:

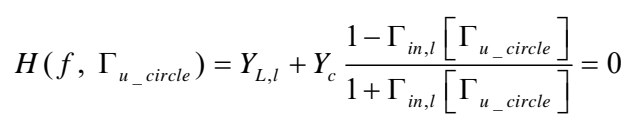


where $\Gamma_{i n, l}\left[\Gamma_{u_{-} \text {circle }}\right]$ indicates the application of (22) to the points belonging to a particular $\Gamma_{u}$ circle (or portion of circle). Equation (24) provides the set of Hopf bifurcation points in the $\Gamma_{l}$ plane, arranged in a manner different from the Hopf loci obtained with (16). Limiting the $\Gamma_{u}$ values considered in (24) to those providing negative resistance at the lower sideband frequency will enable a substantial reduction in computational cost compared with the exhaustive systematic analysis in Section III.

For better insight, instead of applying (24) to the potentially unstable $\Gamma_{u}$ region only, it will be applied to concentric $\Gamma_{u}$ circles, $\Gamma_{u}=\rho e^{j \theta}$, with $\rho$ going from 0 to 1 and $\theta$ going from $0^{\circ}$ to $360^{\circ}$, at different perturbation frequencies. These circles cover the entire Smith chart associated with $\Gamma_{u}$ and will provide the whole geometry of the mapping in (24), including points outside the unit Smith chart corresponding to $\Gamma_{l}$. For each of these sets of $\Gamma_{u}$ circles (corresponding to a particular value of the perturbation frequency $f$ ), another set of circles with their centers on a straight line is obtained in the $\Gamma_{l}$. plane. When varying the perturbation frequency $f$, a collection of circle families is obtained, each for a given value of this frequency.

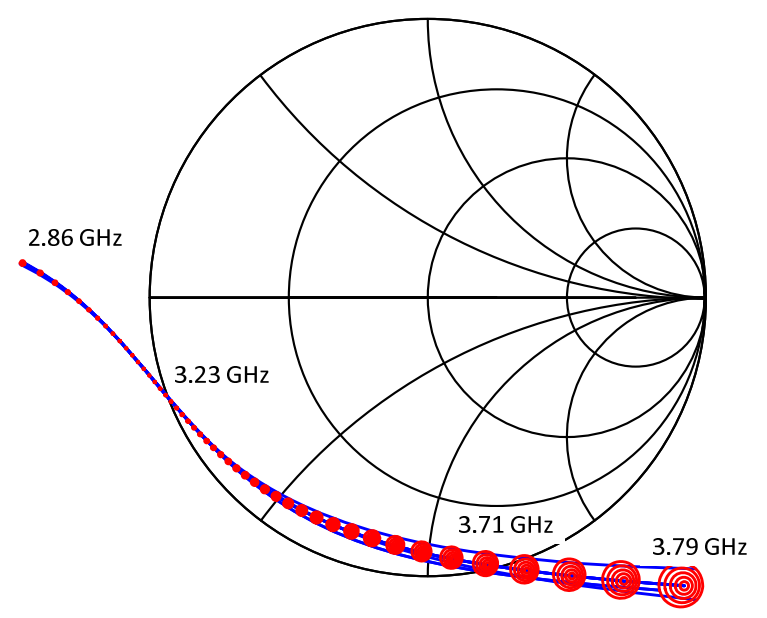

(a)

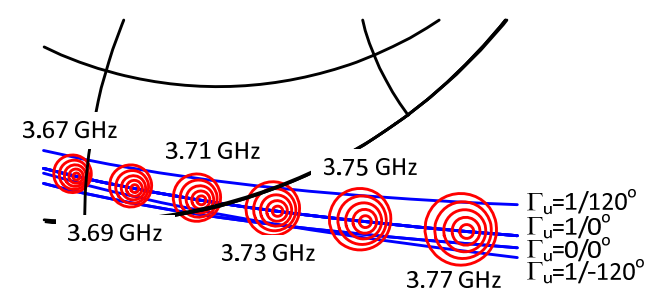

(b)

Fig. 21 Hopf loci points obtained with (24) and with the numerical technique in Section III for constant input power $P_{\text {in }}=-2 \mathrm{dBm}$ for the amplifier in Fig. 10. (a) Full geometry of the circle family. (b) Expanded view.

The above analysis has been applied to the amplifier in Fig. 10 at constant input power $P_{\text {in }}=-2 \mathrm{dBm}$. The points in the resulting circle families (Fig. 21) agree with the points obtained with the numerical technique in Section III, though they are arranged in a different manner. The excellent agreement between these two independent simulations can be noted (Verification VI). Use of the large-signal stability circles provides more clear information on the influence of the reflection coefficients $\Gamma_{o}$ and $\Gamma_{u}$. In this particular case, the influence of $\Gamma_{u}$ is small and increases for smaller value of the perturbation frequency $f$.

\section{CONCLUSIONS}

Several advances in the investigation of instability phenomena in power amplifiers under output mismatch effects have been presented. Instability boundaries are calculated in the output load plane, using concepts from bifurcation theory. The Hopf locus defining the oscillation boundary is obtained by linearizing the circuit either about the $d c$ solution, for a small-signal stability analysis, or the periodic large-signal solution, for a large-signal stability analysis. In the latter case, the linearization is performed with the conversion matrix approach. A detailed study of the geometry of the Hopf bifurcation locus (stability boundary) and its implications on circuit behavior has been presented. In order to evaluate the potential instability of an amplifier in a large-signal regime, a $2 \times 2$ scattering matrix in terms of the two dominant frequency sidebands has been defined. This allows the derivation of conditions for absolute stability in a large-signal regime under output mismatch effects. The stability circles (negativeresistance boundaries) obtained with the $2 \times 2$ scattering matrix are used to obtain the large-signal Hopf bifurcation loci in an efficient manner with a low computational cost. The new formulations and methodologies have been applied to two practical amplifiers, obtaining very good agreement with independent simulations and with measurements.

\section{ACKNOWLEDGEMENT}

Authors are grateful to Dr. Marc Franco from RFMD for bringing the topic of amplifier destabilization under mismatch effects to their attention. Authors are also grateful to José Ángel García from University of Cantabria for helpful discussions.

\section{REFERENCES}

[1] A. Anakabe, N. Ayllon, J.M. Collantes, G. Soubercaze-Pun, and K. Narendra, "Automatic pole-zero identification for multivariable largesignal stability analysis of RF and microwave circuits," European Microwave Conf., Paris, 2010, pp. 477-480.

[2] S. Dellier, R. Gourseyrol, J. Collantes, A. Anakabe, G. SoubercazePun, and K. Narendra, "Stability analysis of microwave circuits," IEEE Wireless and Microwave Techn. Conf. (WAMICON), Cocoa Beach, FL, 2012, pp.1-5.

[3] K. Narendra, E. Limiti, C. Paoloni, J. M. Collantes, R. Jansen, and S. Yarman, "Vectorially Combined Distributed Power Amplifiers for Software-Defined Radio Applications," IEEE Trans. Microw. Theory Techn., vol. 60, no. 10, pp. 3189-3200, Oct., 2012.

[4] J.F. Imbornone, M. Murphy, R.S. Donahue, and E. Heaney, "New insight into subharmonic oscillation mode of GaAs power amplifiers 
Under Severe Output Mismatch Condition," IEEE J. Solid-State Circuits, vol. 32, pp. 1319-1325, Sept., 1997.

[5] D. Qiao, D. Cho, Y. Zhao, T. Hung, D. Kimball, M. Li, and P. Asbeck, "Antenna impedance mismatch measurement and correction for adaptive CDMA transceivers," IEEE MTT-S Int. Microwave Symp., Long Beach, CA, 2005, pp. 783-786.

[6] A. van Bezooijen, C. Chanlo, and A. van Roermund, "Adaptively preserving power amplifier linearity under antenna mismatch," IEEE MTT-S Int. Microwave Symp., Fort Worth, TX, 2004, pp. 1515-1518.

[7] A. Keerti and A. H. Pham, "RF Characterization of SiGe HBT Power Amplifiers Under Load Mismatch," IEEE Trans. Microw. Theory Techn., vol. 55, no. 2, pp. 207-214, Feb., 2007.

[8] W. Karoui and T. Parra, "A protection circuit for HBT RF Power Amplifier under load mismatch conditions," Circuits and Systems and TAISA Conf. - Joint 6th Int. IEEE Northeast Workshop, Montreal, Jun, 2008, pp. 241-244.

[9] A. Suárez, F. Ramírez, and S. Sancho, "Stability analysis of power amplifiers under mismatching effects," IEEE MTT-S Int. Microwave Symp., Seattle, WA, Jun., 2013.

[10] J. Guckenheimer and P. Holmes, Nonlinear oscillations, dynamical systems and bifurcations of vector fields, New York: Springer-Verlag, 1990.

[11] A. Suárez, Analysis and design of autonomous microwave circuits, IEEE-Wiley, Jan. 2009.

[12] V. Rizzoli and A. Neri, "State of the art and present trends in nonlinear microwave CAD techniques," IEEE Trans. Microw. Theory Techn., vol. 36, pp. 343-356, Feb., 1988.

[13] R. Quéré, E. Ngoya, M. Camiade, A. Suarez, M. Hessane, and J. Obregon, "Large signal design of broadband monolithic microwave frequency dividers and phase-locked oscillators," IEEE Trans. Microw. Theory Techn., vol. 41, pp. 1928-1938, Nov., 1993.

[14] A. Suarez, J. Morales, and R. Quéré, "Synchronization analysis of autonomous microwave circuits using new global-stability analysis tools," IEEE Trans. Microw. Theory Techn., vol. 46, no. 5, pp. 494504, May, 1998.

[15] J. M. Paillot, J. C. Nallatamby, M. Hessane, R. Quéré, M. Prigent, and J. Rousset, "A general program for steady state, stability, and FM noise analysis of microwave oscillators," IEEE MTT-S Int. Microwave Symp., Dallas, TX, 1990, pp. 1287-1290.

[16] V. Rizzoli, F. Mastri, and D. Masotti, "General noise analysis of nonlinear microwave circuits by the piecewise harmonic balance technique," IEEE Trans. Microw. Theory Techn., vol. 42, pp. 807-819, May, 1994.

[17] V. Rizzoli and A. Lipparini, "General Stability Analysis of Periodic Steady-State Regimes in Nonlinear Microwave Circuits," IEEE Trans. Microw. Theory Techn., vol. 33, no. 1, pp. 30-37, Jan., 1985.

[18] W. Struble and A. Platzker, "A rigorous yet simple method for determining stability of linear N-port networks [and MMIC application]," Gallium Arsenide Integrated Circuit (GaAs IC) Symp. Techn. Dig., San Jose, CA, 1993, pp. 251-254.

[19] W. Struble and A. Platzker, "Rigorous determination of the stability of linear n-node circuits from network determinants and the appropriate role of the stability factor K of their reduced two-ports," Int. Workshop Integrated Nonlinear Microwave and Millimeterwave Circuits, Duisburg, Oct., 1994, pp. 93-107.

[20] S. Mons, J.-C. Nallatamby, R. Queré, P. Savary, and J. Obregon, "A unified approach for the linear and nonlinear stability analysis of microwave circuits using commercially available tools," IEEE Trans. Microw. Theory Techn., vol. 47, no. 12, pp. 2403-2409, Dec., 1999.

[21] A. Anakabe, J. M. Collantes, J. Portilla, J. Jugo, A. Mallet, L. Lapierre, and J.P. Fraysse, "Analysis and elimination of parametric oscillations in monolithic power amplifiers," IEEE MTT-S Int. Microwave Symp., Seattle, WA, Jun., 2002, pp. 2181-2184.

[22] J. Jugo, J. Portilla, A. Anakabe, A. Suárez, and J. M. Collantes, "Closed-loop stability analysis of microwave amplifiers," IEE Electron. Lett., vol. 37, pp. 226-228, Feb., 2001.
[23] J.M. Collantes, I. Lizarraga, A. Anakabe, and J. Jugo. "Stability verification of microwave circuits through Floquet multiplier analysis," IEEE APCCAS Asia-Pacific Conf. on Circuits Syst., Taiwan, 2004, pp. 997-1000.

[24] F. Bonani and M. Gilli, "Analysis of stability and bifurcations of limit cycles in Chua's circuit through the harmonic-balance approach," IEEE Trans. Circuits Syst. I, vol. 46, pp. 881-890, Aug., 1999.

[25] J.M. Collantes, N. Otegi, A. Anakabe, N. Ayllon, A. Mallet, and G. Soubercaze-Pun, "Monte-Carlo Stability Analysis of Microwave Amplifiers," IEEE Wireless and Microwave Techn. Conf. (WAMICON), Clearwater Beach, FL, 2011, pp.1-6.

[26] S. Jeon, A. Suárez, and R. Rutledge, "Global stability analysis and stabilization of a Class-E/F amplifier with a distributed active transformer," IEEE Trans. Microw. Theory Techn., vol. 53, no. 12, pp. 3712-3722, Dec., 2005.

[27] J. M. Rollett, "Stability and power-gain invariants of linear twoports," Ins. Radio Eng. Trans. Circuit Theory, vol. 9, pp. 29-32, 1962.

[28] E.L. Tan, "Simplified graphical analysis of linear three-port stability," IEE Proc. Microw. Antennas Propag., vol. 152, no. 4, pp. 209-213, Aug., 2005.

[29] J.F. Boehm and W.G. Albright, "Unconditional stability of a three-port network characterized with S-parameters," IEEE Trans. Microw. Theory Techn., vol. 35, no. 6, pp. 582-586, Jun., 1987.

[30] K. Kurokawa, "Some basic characteristics of broadband negative resistance oscillators," The Bell System Technical Journal, vol. 48, pp. 1937-1955, 1969.

[31] R. E. Collin, Foundations for microwave engineering, $2^{\text {nd }}$ ed. John Wiley \& Sons Inc., 2001.

[32] A. Suarez, E. Fernandez, F. Ramirez, and S. Sancho, "Stability and bifurcation analysis of self-oscillating quasi-periodic regimes," IEEE Trans. Microw. Theory Techn., vol. 60, no. 3, pp. 528, 541, Mar., 2012.

[33] C. Kuo, Automatic Control Systems, Prentice Hall, New Jersey, 1995.

[34] N. Ayllon, A. Anakabe, J.M. Collantes, G. Soubercaze-Pun, and S. Forestier, "Sensitivity enhancement in pole-zero identification based stability analysis of microwave circuits," Integrated Nonlinear Microwave and Millimetre-Wave Circuits, Malaga, Nov., 2008., pp.75-78.

[35] F. Di Paolo and G. Leuzzi, "Bifurcation synthesis by means of harmonic balance and conversion matrix," Proc. European Gallium Arsenide Applicat. Symp., Munich, Oct., 2003, pp.521-524.

[36] L. Pantoli, A. Suárez, G. Leuzzi, and F. Di Paolo, "Complete and systematic simulation tools for frequency divider design," IEEE Trans. Microw. Theory Techn., vol. 56, no. 11, pp. 2442-2452, Nov., 2008.

[37] R.M. Foster, "A reactance theorem," Bell Systems Technical Journal, vol. 3, no. 2, pp. 259-267, Nov., 1924

[38] G. D. Vendelin, A. M. Pavio, and U. L. Rohde, Microwave circuit design using linear and nonlinear technique, John Wiley \& Sons, 1990.

[39] M. L. Edwards and J. H. Sinsky, "A new criterion for linear 2-port stability using geometrically derived parameters," IEEE Trans. Microw. Theory Techn., vol. 40, no. 12, pp. 2303-2311, Dec., 1992.

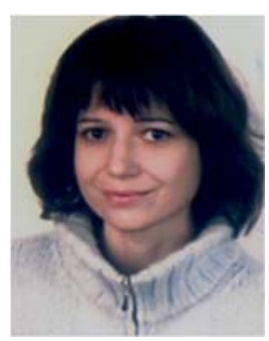

Almudena Suárez was born in Santander, Spain. She received the degree in electronic physics and the Ph.D. degree from the University of Cantabria, Santander, Spain, in 1987 and 1992, respectively, and the Ph.D. degree in electronics from the University of Limoges, France, in 1993. At present, she is a Full Professor at the University of Cantabria, and a member of its Communications Engineering Department. She has authored the book Analysis and design of autonomous microwave circuits for the publisher IEEE-Wiley and co-authored the book Stability analysis of microwave circuits for the publisher ArtechHouse. She belongs to the technical committees of the IEEE International Microwave Symposium and European Microwave 
Conference. She was an IEEE Distinguished Microwave Lecturer for the period 2006-2008. She is a member of the Board of Directors of EuMA and the Editor in Chief of International Journal of Microwave and Wireless Technologies from Cambridge University Press.

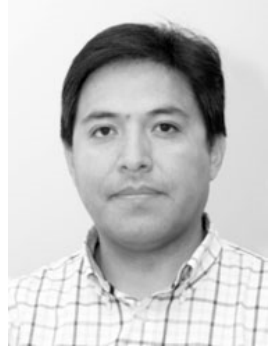

Franco Ramírez was born in Potosí, Bolivia. $\mathrm{He}$ obtained a degree in electronic systems engineering degree from the Military School of Engineering (EMI) in La Paz, Bolivia, in 2000 and the Ph.D. degree in communications engineering from the University of Cantabria, Santander, Spain in 2005. From 1999 to 2000 he worked for Ericsson de Bolivia Telecomunicaciones, where he was involved in several projects related with GSM and TDMA technologies. At present he is an Associate Professor at Dept. of Communications Engineering of the Univ. of Cantabria. His research interests include stability, phase noise, and the development of nonlinear techniques for the analysis and design of autonomous microwave circuits.

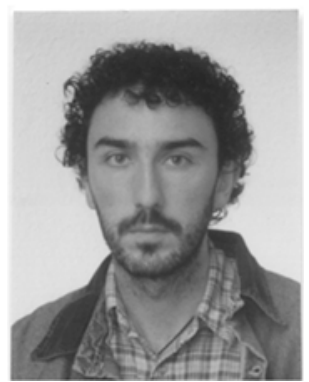

Sergio Sancho was born in Santurce, Spain, in 1973. In 1997 received the degree in Physics from Basque Country University. In 1998 he joined the Communications Engineering Department of the University of Cantabria, Spain, where he received the Ph.D. degree in Electronic Engineering in February 2002. At present, he works at the University of Cantabria, as an Associate Professor of its Communications Engineering Department. His research interests include the nonlinear analysis of microwave autonomous circuits and frequency synthesizers, including stochastic and phase-noise analysis. 